\title{
Reliability Modeling and Analysis of Load- Sharing Systems With Continuously Degrading Components
}

\author{
Xiujie Zhao, Bin Liu, Member, IEEE, and Yiqi Liu
}

\begin{abstract}
This paper presents a reliability modeling and analysis framework for load-sharing systems with identical components subject to continuous degradation. It is assumed that the components in the system suffer from degradation through an additive impact under increased workload caused by consecutive failures. A log-linear link function is used to describe the relationship between the degradation rate and load stress levels. By assuming that the component degradation is well modeled by a step-wise drifted Wiener process, we construct maximum likelihood estimates (MLEs) for unknown parameters and related reliability characteristics by combining analytical and numerical methods. Approximate initial guesses are proposed to lessen the computational burden in numerical estimation. The estimated distribution of MLE is given in the form of multivariate normal distribution with the aid of Fisher information. Alternative confidence intervals are provided by bootstrapping methods. A simulation study with various sample sizes and inspection intervals is presented to analyze the estimation accuracy. Finally, the proposed approach is illustrated by track degradation data from an application example.
\end{abstract}

Index Terms - continuous degradation, data uncertainty, loadsharing system, maximum likelihood estimation, Wiener process.

\section{ACRONYMS}

$\begin{array}{ll}\text { BS } & \text { Bootstrapping } \\ \text { LS } & \text { Large-sample approximation } \\ \text { MLE } & \text { Maximum likelihood estimation } \\ \text { MTTF } & \text { Mean time to failure } \\ \text { SE } & \text { Standard error }\end{array}$

\section{NOTATIONS}

$\begin{array}{ll}B(\cdot) & \text { Standard Brownian motion } \\ J & \text { Number of components in each system } \\ L & \text { Failure threshold }\end{array}$

This work was supported in part by the Research Grants Council of Hong Kong under a theme-based project under Grant T32-101/15-R and a General Research Fund (CityU 11203815) and in part by the National Natural Science Foundation of China under a Key Project under Grant 71532008.

$\mathrm{X}$. Zhao is with the Department of Systems Engineering and Engineering Management, City University of Hong Kong, Kowloon, Hong Kong, and also with the Shenzhen Research Institute, City University of Hong Kong, Shenzhen 518000, China (e-mail: xiujizhao2-c@my.cityu.edu.hk).

\begin{tabular}{|c|c|}
\hline$L_{i j}$ & $\begin{array}{l}\text { Number of degradation inspections for the } \\
j \text { th failed component in the } i \text { th system }\end{array}$ \\
\hline$N$ & Total number of observed systems \\
\hline$w_{j}$ & $\begin{array}{l}\text { Load on each surviving component after the } \\
(j-1) \text { th failure }\end{array}$ \\
\hline$X(t)$ & Wiener degradation process \\
\hline$Y_{i, j}$ & $\begin{array}{l}\text { Lifetime of the } j \text { th failed component in the } \\
i \text { th system }\end{array}$ \\
\hline$\Delta \tau$ & Inspection interval \\
\hline$\Delta \mathrm{x}, \mathrm{y}$ & $\begin{array}{l}\text { Observed degradation increments and fail- } \\
\text { ure time }\end{array}$ \\
\hline$\ell(\boldsymbol{\theta} \mid \Delta \mathbf{x}, \boldsymbol{y})$ & Log-likelihood function \\
\hline$\eta_{j}$ & Drift parameter under load $w_{j}$ \\
\hline$\theta$ & Unknown parameters \\
\hline$\Theta$ & Real space for unknown parameters \\
\hline$\sigma$ & Diffusion parameter \\
\hline$\xi_{j}$ & Standardized stress level under workload $w_{j}$ \\
\hline
\end{tabular}

\section{INTRODUCTION}

$\mathrm{R}$ EDUNDANCY techniques are commonly used to enhance the reliability of various systems. Numerous existing models of reliability redundancy assume that the components are working independently [1]. The assumption of independence provides convenient mathematical properties and computational efficiency in reliability assessment. However, the interdependence of components in redundant systems cannot be ignored for many practical reasons. One typical scenario is that many systems have load-sharing characteristics, i.e., the components are subject to a shared system workload. In such systems, component failures result in an elevated workload of the surviving components, which typically accelerates the failure of the whole system. Load-sharing systems are widely applied in various industries, such as power systems and gear systems [2], [3].

B. Liu is with the Department of Civil and Environmental Engineering, University of Waterloo, Waterloo, ON N2L 3G1, Canada. (b274liu@uwaterloo.ca).

Y. Liu is with the Department of Systems Engineering and Engineering Management, City University of Hong Kong, Kowloon, Hong Kong, and also with the School of Automation Science and Engineering, South China University of Technology, Guangzhou, China (e-mail: aulyq@scut.edu.cn). 
Load-sharing redundant systems have been intensely studied in the literature. Shao and Lamberson [4] presented a Markov model to analyze the $k$-out-of- $n$ load-sharing systems. With respect to such systems, numerous studies have explored the issues of reliability evaluation [5]-[7], inspection scheduling [8], maintenance optimization and system design [9].

To facilitate inspection and maintenance planning, decision makers need to figure out the reliability characteristics of loadsharing systems, which can be modeled by unknown parameters that can be estimated from test or field data. Liu [10] evaluated the reliability of load-sharing $k$-out-of- $n$ systems where the lifetime distributions of the components are different and arbitrary. Kim and Kvam [5] proposed a maximum likelihood estimation (MLE) approach to systems with unknown load-sharing rules. Kvam and Peña [11] used a nonparametric method to make inferences of load-sharing life models. Park [12] considered a parallel load-sharing system with identical components and derived analytical MLEs by assuming that the underlying lifetime distribution of each component is exponential or Weibull. In a follow-up study [13], Expectation-Maximization (EM) algorithm was adopted to estimate the parameters for similar systems with components of which lifetime distribution is lognormal or normal. Aside from these, interval estimation for the reliability of $k$-out-of- $n$ load-sharing systems were studied with exponential component lifetime [7]. Wang et al. [14] evaluated the reliability of load-sharing parallel systems by introducing the failure dependency and characterized the system dynamics with the semi-Markov process. However, most of these studies have focused on the lifetime modeling of load-sharing systems, where only shock failures were considered.

As sensor technologies advance rapidly, the degradation of quality characteristics (QC) of many systems can be observed and measured precisely. System degradation has been proven to be closely associated with reliability. For systems suffering from corrosion, wear or cumulative usage, degradation measures provide reasonable predictions of system failures. For some other systems, the degradation can be measured by the performance reduction. For instance, LED lamps and LCD monitors are deemed to have failed when the brightness falls below a critical level. Stochastic process models and general path models are two main types of degradation modeling approaches. The most widely used stochastic processes to model degradation data include Wiener process [15], [16], gamma process [17] and inverse Gaussian process [18]. Stochastic models have clear physical explanations, making it convenient to incorporate covariates and random effects to reflect various properties of degradation data. For general path models, Hong et al. [19] modeled the degradation of an organic coating in environments with dynamic covariates. In an extended work by Xu et al. [20], nonlinear general path models with time-varying environmental covariates were analyzed.

Although degradation-based models are considered to be superior in reliability analysis, there is hardly any literature addressing the reliability of load-sharing systems with degrading components. Ye et al. [21] proposed the cumulative workload (CWL) to degradation failure mode to model the load-sharing system and carried out a cost analysis. Liu et al. [22] presented a preventive maintenance modeling approach to load-sharing parallel systems with identical degrading components. In Liu et al. [23], the MLE of parameters of load-sharing systems was discussed for Wiener processes and inverse Gaussian processes. Nevertheless, the assumption of different parameters for different workloads adopted in Liu et al. [23] and many previous works [12], [13] makes the statistical inference less efficient as the number of components in the system increases. Moreover, to the best of our knowledge, no literature addressed the variability of parameter estimates for degrading loadsharing system. This study intends to fill this gap.

In this paper, we present systematic parameter estimation procedures for parallel load-sharing systems with continuously degrading components. First, we construct the system reliability model and identify unknown parameters. The components in the system are assumed either identical or heterogeneous. Wiener process is used to model the degradation path of each component. We assume that the system load is evenly distributed to each working component. To reduce the number of unknown parameters, we take advantage of a link function that describes the relationship between the degradation rate and the workload. Afterward, the MLEs of unknown parameters are obtained by numerical methods. Finally, we use two methods to quantify the uncertainty in parameter estimates. The largesample approximation method gives the Fisher information and constructs the estimated joint distribution for parameter estimates to allow interval estimation. Alternatively, bootstrapping approach can generate a large sample of parameter estimates to quantify the estimation variability nonparametrically.

The remainder of the paper is organized as follows. Section II presents the reliability modeling of load-sharing systems with degrading components. In Section III, the likelihood function is formulated and the estimated distribution of unknown parameters is derived. A simulated numerical example is used to illustrate the proposed method in Section IV. Section V presents a case study with data from a track degradation test. Finally, Section VI gives concluding remarks and suggestions for future works.

\section{DESCRIPTION AND MODELING OF LOAD-SHARING SYSTEMS WITH DEGRADING COMPONENTS}

Components in load-sharing systems generally have dependent degradation paths due to the common system load imposed upon them. At the time when the system initiates to work, the components degrade slowly as the load on each component is low. When the degradation levels of some components in the system reach the critical failure threshold, these components are deemed to have failed. In other words, they are not able to share the system load afterward. In this situation, each surviving component in the system has to burden heavier workload and thereby suffer from higher degradation rates. An illustrative degradation path of such a system is shown in Fig. 1. One typical example of such systems comes from the railway systems. For particular areas of the track where vehicles frequently go by, when the wear of some subsections becomes severe, the wheels have no seamless contact on these subsections. Meanwhile, other small subsections of the track tend to suffer a higher rate of wear afterward. Another example that has the similar load-sharing mechanism is the wastewater treatment system. Activated sludge process (ASP) is the most commonly used technique to remove organic matter and nutrients (mainly 


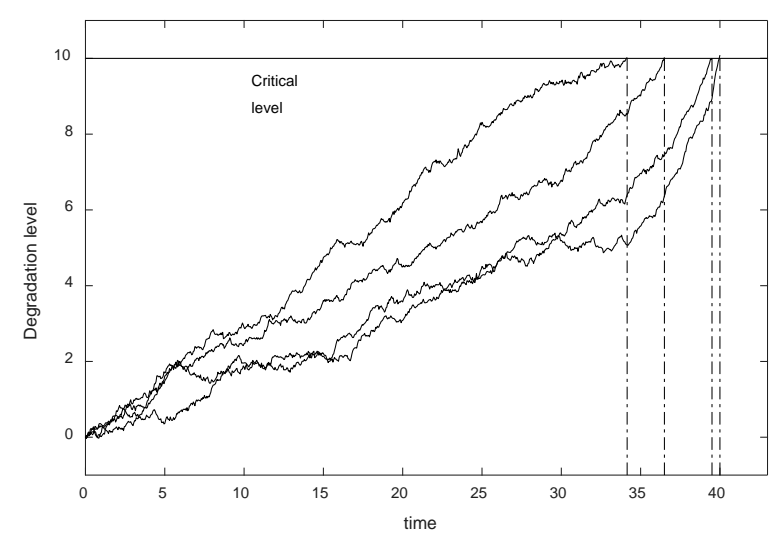

Fig. 1. An illustration of degradation levels of load-sharing systems with four parallel components

nitrogen and phosphorus) in the wastewater plant [24]. During the organic matter and nutrients degradation process, several aerobic biological tanks are connected to each other to ensure the treatment efficiency. However, once the treatment efficiency of an aerobic biological system reaches the critical failure threshold, the corresponding component fails and it cannot share the workload afterward. This makes other aerobic tanks burden heavier workload and this accelerates their degradation processes.

\section{A. Assumptions and Wiener Degradation Models}

Consider $N$ identical systems each with $J$ components connected in parallel. For each system, we make the following assumptions that are similar with those in Park [13]:

1. Each component is subject to continuous degradation that can be well modeled by a Wiener process.

2. Each component is deemed to have failed when its degradation level exceeds a predetermined threshold $L$.

3. The load of the whole system is constant and is equally distributed to each working component.

4. The degradation measures are taken periodically.

5. Component failures are self-announcing, i.e., the exact failure time of each component can be observed.

Remark: Assumption 5 is made based on real practices in reliability tests. Component failures are commonly easier to observe than degradation levels. For example, in adhesive bond tests [25] the failures are immediately observed as the bonds break up upon failures. However, the degradation level of adhesive bonds cannot be observed continuously. Engineers need to employ specific instruments to measure the degradation level. Another example is from water treatment systems consisting of multiple filters. When a filter degrades to the critical level, it cannot yield the required volume of water, which can be detected immediately. In contrast, the degradation levels of these filters need to be revealed by inspections, which are usually carried out periodically.

When all $J$ components in the system are working, we use a linear Wiener process $X(t)$ to model the degradation process for each component, that is

$$
X(t)=\eta_{1} t+\sigma B(t)
$$

where $\eta_{1}$ is the drift parameter, $\sigma$ is the diffusion parameter, and $B(\cdot)$ is the standard Brownian motion. For any component in the system, the lifetime follows an inverse Gaussian distribution with mean $L / \eta_{1}$ and shape $L^{2} / \sigma^{2}$, and the distribution function is denoted by $F_{\mathcal{J G}}\left(t ; L / \eta_{1}, L^{2} / \sigma^{2}\right)$.

\section{B. Load-Sharing Modeling and Link Function}

Since the component failure times are s-dependent in a loadsharing system, it is inappropriate to model the degradation process for each component independently. Under the assumption that the components in the systems are of the same type and the workload on each component is equal, it is reasonable to imply that each surviving component is suffering from an equal damage that leads to degradation growth at an arbitrary time.

Let $Y_{i, j}, j=1, \ldots, J$ be the time at which the $j$ th component in the $i$ th system fails, where $Y_{i, 1} \leq \cdots \leq Y_{i, J}, i=$ $1, \ldots, N$. Note that components $j=1, \ldots, J$ are ordered by the sequence of failures. A realization of $Y_{i, j}$ is denoted by $y_{i, j}$. Specifically, we assume that $Y_{i, 0} \equiv 0$ and $y_{i, 0} \equiv 0$. For simplicity, we denote the $j$ th component in system $i$ by component $(i, j)$ in the following contexts.

For period $Y_{i, j-1}<t \leq Y_{i, j}$, the workload on each component is denoted by $w_{j}$. If the total load is normalized as 1 , it is straightforward that $w_{j}=1 /(J-j+1)$ for $j=1, \ldots, J$. In other words, the last failing component experiences $J$ different workloads throughout the lifespan of the system. Under $w_{j}$, we assume that the Wiener degradation parameters for a single surviving component are $\eta_{j}$ and $\sigma_{j}$. Some previous studies [13], [26] have assumed an additive parameter under each load and estimated the parameters. However, in many real parallel systems, the number of components may be relatively large, and this approach will introduce a large number of unknown parameters, which deteriorates the generality and efficiency of statistical inferences. As stated in Kong and Ye [7], we can resort to establishing a link function to connect the workload and degradation model parameters for degrading components in loadsharing systems.

As stated in the literature that discussed the relationship between the Wiener degradation model and external stresses [27], [28], it is reasonable to assume that the diffusion parameter $\sigma$ does not change across various workloads and environments, i.e., $\sigma_{j} \equiv \sigma$. The assumption of constant diffusion parameter has been widely validated by many real degrading products, such as LED lamps [29] and carbon-film resistors [30]. A loglinear link function for $\eta_{j}$ is assumed as follows:

$$
\log \left(\eta_{j}\right)=\beta_{0}+\beta_{1} \xi_{j}, \quad j=1, \ldots, J .
$$

Let $\boldsymbol{\xi}=\left(\xi_{1}, \ldots, \xi_{J}\right)^{\prime}$, where $\xi_{j}=\xi\left(w_{j}\right)$ is the standardized stress level under workload $w_{j}$, and we have $0=\xi_{1}<\cdots<$ $\xi_{J}=1$. The form of $\xi\left(w_{j}\right)$ varies for different types of systems and loads, and it is noted that there are typically no unknown parameters in $\xi\left(w_{i}\right)$. In reliability analysis, the log-linear link functions are commonly used in degradation modeling and accelerated tests [25], [31]. Further discussions of the log-linear link function and $\xi\left(w_{i}\right)$ can be found in Appendix A. 


\section{Reliability Function}

We use random vector $\boldsymbol{Y}_{i}=\left(Y_{i, 1}, \ldots, Y_{i, J}\right)^{\prime}$ to denote the failure time of each component in an arbitrary system $i$, where $Y_{i, 1} \leq \cdots \leq Y_{i, J}$. The distribution function for the failure time of the whole system is represented in a conditional manner as follows:

$$
\begin{aligned}
F(t) & =\operatorname{Pr}\left(Y_{i, J} \leq t\right) \\
=\int_{y_{J-1}}^{t} \ldots \int_{y_{2}}^{t} \int_{y_{1}}^{t} & \int_{0}^{t} f_{Y_{J}}\left(y_{J} \mid Y_{i, 1}=y_{1}, \ldots, Y_{i, J-1}\right. \\
& \left.=y_{J-1}\right) \ldots f_{Y_{i, 2}}\left(y_{2} \mid Y_{i, 1}\right. \\
& \left.=y_{1}\right) f_{Y_{i, 1}}\left(y_{1}\right) \mathrm{d} y_{1} \mathrm{~d} y_{2} \ldots \mathrm{d} y_{J}
\end{aligned}
$$

where $f_{Y_{i, 1}}(\cdot)$ is the density function of $Y_{i, 1}$, and $f_{Y_{i, j}}(\cdot \mid \cdot)$ is the conditional density of $Y_{i, j}$ for $j=2, \ldots, J$. Specifically, since $Y_{i, 1}$ is the first order statistic, the distribution function of $Y_{i, 1}$ evaluated at $Y_{i, 1}=y_{1}$ is given by the probability that the minimum of the first passage times (FPTs) of all $J$ degradation processes initiating at zero is smaller than $y_{1}$. As the degradation processes are mutually independent between any two consecutive failures, we can obtain that $F_{Y_{i, 1}}\left(y_{1}\right)=1-[1-$ $\left.F_{\mathcal{J G}}\left(y_{1} ; L / \eta, L^{2} / \sigma^{2}\right)\right]^{J}$, and the density function of $Y_{i, 1}$ is

$$
\begin{aligned}
& f_{Y_{i, 1}}\left(y_{1}\right)=\frac{\partial F_{Y_{i, 1}}\left(y_{1}\right)}{\partial y_{1}} \\
& =\frac{\partial\left[1-\left(1-F_{\mathcal{J G}}\left(y_{1} ; L / \eta_{1}, L^{2} / \sigma^{2}\right)\right)^{J}\right]}{\partial y_{1}} \\
& =J\left[1-F_{\mathcal{J} \mathcal{G}}\left(y_{1} ; L / \eta_{1}, L^{2} / \sigma^{2}\right)\right]^{J-1} f_{\mathcal{J} \mathcal{G}}\left(y_{1} ; L / \eta_{1}, L^{2} / \sigma^{2}\right)
\end{aligned}
$$

Further, the conditional density for $Y_{i, j}, j \geq 2$ is given by

$$
\begin{aligned}
& f_{Y_{i, j}}\left(y_{j} \mid Y_{i, 1}=y_{1}, \ldots, Y_{i, j-1}=y_{j-1}\right) \\
& \approx \int_{0}^{L} \ldots \int_{0}^{L} f_{\mathcal{T} \mathcal{N}}\left(x_{1} ; Y_{i, 1}=y_{1}, \ldots, Y_{i, j-1}=y_{j-1}\right) \times \\
& \quad \ldots \times f_{\mathcal{T} \mathcal{N}}\left(x_{J-j+1} ; Y_{i, 1}=y_{1}, \ldots, Y_{i, j-1}=y_{j-1}\right) \times \\
& \quad \times f_{Y_{i, j}}\left(y_{j} \mid x_{1}, \ldots, x_{J-j+1}, y_{j-1}\right) \mathrm{d} x_{1} \ldots \mathrm{d} x_{J-j+1},
\end{aligned}
$$

and $f_{\mathcal{T} \mathcal{N}}\left(x ; Y_{i, 1}=y_{1}, \ldots, Y_{i, j-1}=y_{j-1}\right)$ can be given by

$$
\begin{aligned}
& f_{\mathcal{T N}}\left(x ; Y_{i, 1}=y_{1}, \ldots, Y_{i, j-1}=y_{j-1}\right) \\
& =f_{\mathcal{T N}}\left(x ; \sum_{j^{\prime}=1}^{j-1} \eta_{j^{\prime}}\left(y_{j^{\prime}}-y_{j^{\prime}-1}\right), y_{j-1} \sigma^{2}, 0, L\right)
\end{aligned}
$$

and $f_{\mathcal{T} \mathcal{N}}\left(x ; \mu, \sigma^{2}, a, b\right)$ is the density function of the truncated normal distribution with mean $\mu$ and variance $\sigma^{2}$, and upper and lower bounds being $a$ and $b$, respectively. Likewise, in (4), the conditional density $f_{Y_{i, j}}\left(y_{j} \mid x_{1}, \ldots, x_{J-j+1}\right)$ is given by (7).

We can derive the reliability function at a given time via (3) by $R(t)=1-F(t)$. Numerical evaluation can be carried out by utilizing (3)-(7). However, the evaluation is very computationally intensive due to the multiple integrals. In Appendix B, we use an approximation-based simulation method to generate samples of the failure time, then the reliability function can be evaluated non-parametrically via simulated life data.

\section{DATA MODELING AND ESTIMATION OF UNKNOWN PARAMETERS}

\section{A. Data Modeling and Contributions to Likelihood}

In this study, we assume that periodic inspections are carried out on each surviving component for system $i$ where $i=$ $1, \ldots, N$, and the inspection interval is fixed at $\Delta \tau$. Denote the number of degradation inspections for component $(i, j)$ by $L_{i j}$, then we have $L_{i j}=\left\lfloor Y_{i, j} / \Delta \tau\right\rfloor$. For component $(i, j)$, let $X_{i j k}$ be the $k$ th degradation measurement, where $k=1, \ldots, L_{i j}$. The measured degradation increments for component $(i, j)$ are denoted by $\Delta \boldsymbol{X}_{i j}=\left(\Delta X_{i j 1}, \ldots, \Delta X_{i j L_{i j}}\right)^{\prime}$, of which each element is given by $\Delta X_{i j k}=X_{i j k}-X_{i j(k-1)}$. Note that we set $X_{i j 0} \equiv 0$. Let $M_{i j}=Y_{i j}-L_{i j} \Delta \tau$ be the time to failure since the final inspection for component $(i, j)$. If $L_{i j} \geq 1$, for $2 \leq j^{\prime} \leq j, \Delta X_{i j k}$ follows normal distributions as shown in (8). Here, we note that it is likely that more than one component in the system fails between two particular inspection epochs. In this paper, we assume that $\Delta \tau$ is relatively small so that the chance of such cases of multiple failures is low. Additionally, even though few such cases occurred, the normal distribution in (8) gives a good approximation for the degradation increments. If a dataset contains a considerable number of cases where several failures occur in one inspection interval for one system, we can change the mean of the normal distribution in the third case

$$
f_{Y_{i, j}}\left(y_{j} \mid x_{1}, \ldots, x_{J-j+1}, y_{j-1}\right)=\frac{\partial\left\{1-\prod_{j^{\prime}=1}^{J-j+1}\left[1-F_{\mathcal{J G}}\left(y_{j}-y_{j-1} ;\left(L-x_{j^{\prime}}\right) / \eta,\left(L-x_{j^{\prime}}\right)^{2} / \sigma^{2}\right)\right]\right\}}{\partial y_{j}} .
$$

$$
\Delta X_{i j k^{\sim}} \sim \begin{cases}\mathcal{N}\left(\eta_{1} \Delta \tau, \sigma^{2} \Delta \tau\right), & \text { if } 1 \leq k \leq L_{i 1}, \\ \mathcal{N}\left(\eta_{j^{\prime}} \Delta \tau, \sigma^{2} \Delta \tau\right), & \text { if } L_{i\left(j^{\prime}-1\right)}+2 \leq k \leq L_{i j^{\prime}}, \\ \mathcal{N}\left(M_{i\left(j^{\prime}-1\right)} \eta_{j^{\prime}-1}+\eta_{j^{\prime}}\left(\Delta \tau-M_{i\left(j^{\prime}-1\right)}\right), \sigma^{2} \Delta \tau\right), & \text { if } k=L_{i\left(j^{\prime}-1\right)}+1\end{cases}
$$


in (8) into a linear combination of more than two piecewise degradation models with change points. The details are given in Appendix C. For simplicity, we employ (8) to model the increments in the following context.

By utilizing the independence of non-overlapping increments of Wiener process, we can evaluate the likelihood contribution of $\Delta \boldsymbol{X}_{i j}$ conveniently by computing the product of likelihoods contributed by $\Delta X_{i j k}$ for all $k$. Furthermore, the observed information provided by component $(i, j)$ also contains $Y_{i, j}$. In other words, the FPT of the degradation process to the critical level $L$ is $Y_{i, j}$. Since the FPT of a Wiener process follows the inverse Gaussian distribution, by conditioning on observing the last degradation measure $X_{i j L_{i j}}=x$, the FPT beyond the last inspection $M_{i j} \sim \mathcal{J} \mathcal{G}\left((L-x) / \eta_{j},(L-x)^{2} / \sigma^{2}\right)$, and the density function is given by

$$
\begin{aligned}
& f_{M_{i j}}\left(m ; L, x, \eta_{j}, \sigma\right) \\
& =\left[\frac{(L-x)^{2}}{2 \pi \sigma^{2} m^{3}}\right]^{1 / 2} \exp \left\{\frac{-\left(\eta_{j} m-L+x\right)^{2}}{2 \sigma^{2} m}\right\} .
\end{aligned}
$$

If component $(i, j)$ fails before any degradation measure is taken, i.e., $L_{i j}=0$, the likelihood is merely contributed by $M_{i j}=Y_{i, j}$.
In the model we have described, the unknown parameters can be denoted by $\boldsymbol{\theta}=\left(\beta_{0}, \beta_{1}, \sigma\right)^{\prime}$. Let $\boldsymbol{\Delta} \mathbf{x}, \boldsymbol{y}$ and $\boldsymbol{l}$ be the realizations of $\Delta \mathbf{X}=\left\{\Delta \boldsymbol{X}_{i j}, i=1, \ldots, N, j=1, \ldots, J\right\}, Y_{i, j}$ and $L_{i j}$ for all the components in all the systems, and $l_{i j}$ is equal to the length of $\Delta \mathbf{x}_{i j}$. Based on (8) and (9), we can evaluate the total likelihood with (10).

To obtain the MLEs of the unknown parameters, we need to rewrite the likelihood function into the log-likelihood function as shown in (11). Since the log-likelihood function is complicated and the link function is nonlinear, it is very difficult, if not impossible, to obtain closed-form MLEs by directly taking first derivatives of $\ell(\boldsymbol{\theta} \mid \boldsymbol{\Delta} \mathbf{x}, \mathbf{y})$. Alternatively, we resort to numerical methods to maximize the log-likelihood function. Newton or quasi-Newton optimization methods [32] have been widely used to solve non-linear programming problems and they can be easily implemented in various software packages for numerical analysis and optimization.

\section{B. Initial Guesses in Parameter Estimation}

The efficiency of Newton optimization method depends on the initial guess to a great extent. A better initial point can significantly decrease the number of iterations till convergence, especially when the sample size is relatively large. Therefore, we propose to make an initial guess that is reasonably close to the MLE of $\boldsymbol{\theta}$ to facilitate the estimation procedure. Partial observations from the complete dataset are used to rapidly generate initial guesses. Specifically, we use part of the degradation measurements $\Delta \mathrm{x}$ to obtain rough estimates of $\boldsymbol{\theta}$. First, for

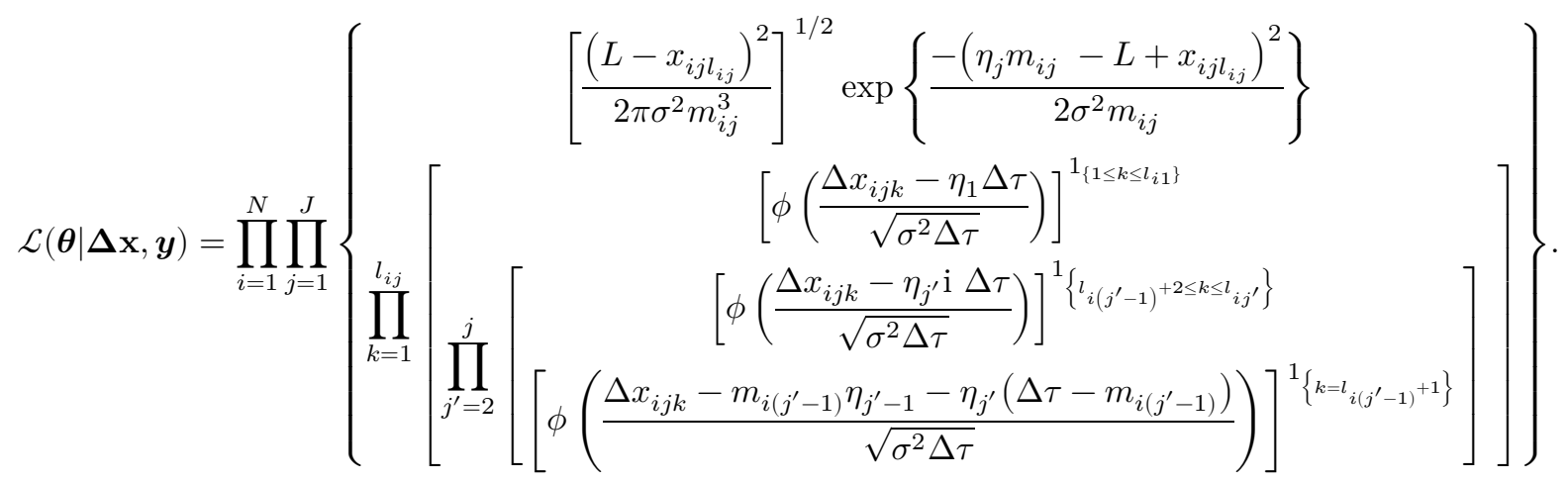

$$
\begin{aligned}
\ell(\boldsymbol{\theta} \mid \boldsymbol{\Delta} \mathbf{x}, \boldsymbol{y})=\sum_{i=1}^{N} & \sum_{j=1}^{J}\left\{\log \left(L-x_{i j l_{i j}}\right)-\frac{1}{2} \log (2 \pi)-\log \sigma-\frac{3}{2} \log m_{i j}-\frac{\left(\eta_{j} m_{i j}-L+x_{i j l_{i j}}\right)^{2}}{2 \sigma^{2} m_{i j}}\right. \\
& +\sum_{k=1}^{l_{i j}}\left\{-\frac{1}{2} \log 2 \pi-\log \sigma-1_{\left\{1 \leq k \leq l_{i 1}\right\}} \frac{\left(\Delta x_{i j k}-\eta_{1} \Delta \tau\right)^{2}}{2 \sigma^{2} \Delta \tau}\right. \\
& -\sum_{j^{\prime}=2}^{j}\left\{1_{\left\{l_{i\left(j^{\prime}-1\right)}+2 \leq k \leq l_{i j^{\prime}}\right\}} \frac{\left(\Delta x_{i j k}-\eta_{j^{\prime}} \Delta \tau\right)^{2}}{2 \sigma^{2} \Delta \tau}\right. \\
& \left.\left.\left.+1_{\left\{k=l_{i\left(j^{\prime}-1\right)}+1\right\}} \frac{\left(\Delta x_{i j k}-m_{i\left(j^{\prime}-1\right)} \eta_{j^{\prime}-1}-\eta_{j^{\prime}}\left(\Delta \tau-m_{i\left(j^{\prime}-1\right)}\right)\right)^{2}}{2 \sigma^{2} \Delta \tau}\right\}\right\}\right\} .
\end{aligned}
$$


system $i$, assume that component $(i, 1)$ fails after one or more degradation measurements, then for $1 \leq l<l_{i 1}$ and components $(i, 1), \ldots(i, J)$, we can obtain an approximated estimate of $\eta_{1}$ by minimizing the mean square error (MSE):

$$
\mathrm{MSE}_{1}=\sum_{i=1}^{N} \sum_{j^{\prime}=1}^{J} \sum_{k=1}^{l_{i 1}}\left(\Delta x_{i j^{\prime} k}-\eta_{1} \Delta \tau\right)^{2}
$$

and let the rough estimate of $\eta_{1}$ be $\tilde{\eta}_{1}$. Similarly, for any $\eta_{j}, j=$ $2, \ldots, J$, we can obtain the estimate $\tilde{\eta}_{j}$ by minimizing the following MSE:

$$
\mathrm{MSE}_{j}=\sum_{i=1}^{N} \sum_{j^{\prime}=j}^{J} \sum_{k=l_{i\left(j^{\prime}-1\right)}+1}^{l_{i j^{\prime}}}\left(\Delta x_{i j^{\prime} k}-\eta_{j} \Delta \tau\right)^{2} .
$$

Note that the minimization of (12) and (13) can be simply realized by the mean increments under a particular load stress divided by $\Delta \tau$. Thus, an estimated vector of drift parameters under different load stresses can be obtained and denoted by $\tilde{\boldsymbol{\eta}}=\left(\tilde{\eta}_{1}, \ldots, \tilde{\eta}_{J}\right)^{\prime}$. As with the link function defined in (2), we compute the initial estimates of $\beta_{0}$ and $\beta_{1}$ by fitting a linear regression model as follows:

$$
\log (\tilde{\boldsymbol{\eta}})=\mathbf{1}_{J} \beta_{0}+\boldsymbol{\xi} \beta_{1}+\boldsymbol{\epsilon},
$$

where $\mathbf{1}_{J}$ is the $J$-dimensional column vector with all elements equal to 1 . From the linear regression, we can easily compute least square estimates of $\beta_{0}$ and $\beta_{1}$, denoted by $\tilde{\beta}_{0}$ and $\tilde{\beta}_{1}$, respectively. Finally, we plug $\tilde{\beta}_{0}$ and $\tilde{\beta}_{1}$ into (6) and maximize $\ell\left(\sigma \mid \boldsymbol{\Delta} \mathbf{x}, \boldsymbol{y}, \boldsymbol{\xi}, \tilde{\beta}_{0}, \tilde{\beta}_{1}\right)$ to get the initial estimate for the diffusion parameter, i.e., $\tilde{\sigma}$. The maximization is straightforward and numerically simple as there is only one unknown variable. Through the above steps, the initial starting point to maximize (11) is determined. From the initial guess, with the aid of numerical optimization packages, the MLE of $\boldsymbol{\theta}$ is computed by

\section{Estimated Distribution of Unknown Parameters}

Rather than use point estimates to support decision making, engineers usually prefer interval estimates of unknown parameters to capture the data uncertainty, thereby better support future decisions and evaluate potential risks. Under comparatively large samples, a multivariate normal (MVN) distribution provides a satisfactory approximation for the joint distribution of the MLEs. In a degrading load-sharing system, owing to the measurable degradation characteristics, the system can provide much more data in addition to failure time data. Thus, the observations from a limited number of systems can contribute to a relatively large sample size, making the MVN approximation reasonably accurate. Let $\hat{\boldsymbol{\theta}}$ be the MLE of $\boldsymbol{\theta}$, the distribution of $\hat{\boldsymbol{\theta}}$ can be approximated by a MVN, i.e., $\widehat{\boldsymbol{\theta}} \sim \mathcal{N}\left(\boldsymbol{\theta},[\mathbf{I}(\boldsymbol{\theta})]^{-\mathbf{1}}\right)$, where $\mathbf{I}(\boldsymbol{\theta})$ is the Fisher information matrix evaluated at $\boldsymbol{\theta}$, which can be evaluated by (15), where $\mathbb{E}[\mathcal{F}]$ is the expected information provided by each single system. The expectation of second derivative functions of $\ell(\boldsymbol{\theta} \mid \boldsymbol{\Delta} \mathbf{x}, \boldsymbol{y})$ is difficult to express in closed forms because we have $\Delta \mathrm{x}$ and $\boldsymbol{y}$ as two dependent sources of data. Moreover, the number of observations in $\Delta \mathrm{x}$ is random. Alternatively, by letting $\Delta \mathrm{X}_{\mathrm{S}}$ and $\boldsymbol{Y}_{\mathrm{S}}$ denote the observed data from one single system, we use the conditional methods to compute the expectation by the following rule:

$$
\mathbb{E}[\mathcal{F}]=\mathbb{E}_{\boldsymbol{Y}_{\mathrm{S}}}\left\{\mathbb{E}_{\boldsymbol{\Delta}} \mathbf{X}_{\mathrm{S}}\left[\mathcal{F} \mid \boldsymbol{Y}_{\mathrm{S}}=\boldsymbol{y}_{\mathrm{S}}\right]\right\}
$$

where $\boldsymbol{y}_{\mathrm{S}}=\left(y_{1}, \ldots, y_{J}\right)^{\prime}$ is a realization of component failure times in ascending order for a single system, $m_{j}$ and $l_{j}$ can be computed by $\boldsymbol{y}_{\mathrm{S}}$ for $j=1, \ldots, J$. The elements in the conditional expectation $\mathbb{E}_{\Delta \mathbf{X}_{\mathrm{S}}}\left[\mathcal{F} \mid \boldsymbol{Y}_{\mathrm{S}}=\boldsymbol{y}_{\mathrm{S}}\right]$ can be derived in closed form. Let

$$
\mathcal{F}=\left[\begin{array}{ccc}
\mathcal{F}_{11} & \mathcal{F}_{12} & 0 \\
\mathcal{F}_{12} & \mathcal{F}_{22} & 0 \\
0 & 0 & \mathcal{F}_{33}
\end{array}\right]
$$

and

$$
\widehat{\boldsymbol{\theta}}=\arg _{\boldsymbol{\theta}} \max \{\ell(\boldsymbol{\theta} \mid \boldsymbol{\Delta} \mathbf{x}, \mathbf{y}) ; \boldsymbol{\theta} \in \boldsymbol{\Theta}\} .
$$

$$
\begin{aligned}
& \mathbf{I}(\boldsymbol{\theta})=\left[\begin{array}{ccc}
\mathbb{E}\left[-\frac{\partial^{2} \ell(\boldsymbol{\theta} \mid \boldsymbol{\Delta} \mathbf{x}, \boldsymbol{y})}{\partial \beta_{0}^{2}}\right] & \mathbb{E}\left[-\frac{\partial^{2} \ell(\boldsymbol{\theta} \mid \boldsymbol{\Delta} \mathbf{x}, \boldsymbol{y})}{\partial \beta_{0} \partial \beta_{1}}\right] & 0 \\
\mathbb{E}\left[-\frac{\partial^{2} \ell(\boldsymbol{\theta} \mid \boldsymbol{\Delta} \mathbf{x}, \boldsymbol{y})}{\partial \beta_{1} \partial \beta_{0}}\right] & \mathbb{E}\left[-\frac{\partial^{2} \ell(\boldsymbol{\theta} \mid \boldsymbol{\Delta} \mathbf{x}, \boldsymbol{y})}{\partial \beta_{0}^{2}}\right] & 0 \\
0 & 0 & \mathbb{E}\left[-\frac{\partial^{2} \ell(\boldsymbol{\theta} \mid \boldsymbol{\Delta} \mathbf{x}, \boldsymbol{y})}{\partial \sigma^{2}}\right]
\end{array}\right] \\
& =N \mathbb{E}\left\{\left[\begin{array}{ccc}
\mathcal{F}_{11} & \mathcal{F}_{12} & 0 \\
\mathcal{F}_{21} & \mathcal{F}_{11} & 0 \\
0 & 0 & \mathcal{F}_{33}
\end{array}\right]\right\}=N \mathbb{E}[\mathcal{F}],
\end{aligned}
$$




$$
\begin{aligned}
\mathbb{E}_{\boldsymbol{\Delta} \mathbf{X}_{\mathrm{S}}\left[\mathcal{F}_{11} \mid \boldsymbol{Y}_{\mathrm{S}}=\right.} & \left.\boldsymbol{y}_{\mathrm{S}}\right] \\
& =\frac{1}{\sigma^{2}} \sum_{j=1}^{J}\left\{\eta_{j}^{2} m_{j}+l_{j} \eta_{1}^{2} \Delta \tau\right. \\
& +1_{\{j \geq 2\}} \sum_{j^{\prime}=2}^{j}\left\{\max \left\{l_{j^{\prime}}-l_{j^{\prime}-1}, 0\right\} \eta_{j^{\prime}}^{2} \Delta \tau\right. \\
& \left.\left.+\frac{\left.\left[m_{j^{\prime}-1} \eta_{j^{\prime}-1}+\eta_{j^{\prime}}\left(\Delta \tau-m_{j^{\prime}-1}\right)\right]^{2}\right\}}{\Delta \tau}\right\}\right\} \\
& =\frac{1}{\sigma^{2}} \sum_{j=1}^{J} \mathbb{E}_{\Delta \mathbf{x}_{\mathrm{S}}}\left[\mathcal{F}_{j} \mid \boldsymbol{Y}_{\mathrm{S}}=\boldsymbol{y}_{\mathrm{S}}\right],
\end{aligned}
$$

and

$$
\begin{aligned}
& \mathbb{E}_{\boldsymbol{\Delta} \mathbf{X}_{\mathrm{S}}}\left[\mathcal{F}_{12} \mid \boldsymbol{Y}_{\mathrm{S}}=\boldsymbol{y}_{\mathrm{S}}\right]=\frac{1}{\sigma^{2}} \sum_{j=1}^{J} \xi_{j} \mathbb{E}_{\Delta \mathrm{x}_{\mathrm{S}}}\left[\mathcal{F}_{j} \mid \boldsymbol{Y}_{\mathrm{S}}=\boldsymbol{y}_{\mathrm{S}}\right] \text {, } \\
& \mathbb{E}_{\Delta \mathbf{x}_{\mathrm{S}}}\left[\mathcal{F}_{22} \mid \boldsymbol{Y}_{\mathrm{S}}=\boldsymbol{y}_{\mathrm{S}}\right]=\frac{1}{\sigma^{2}} \sum_{j=1}^{J} \xi_{j}^{2} \mathbb{E}_{\Delta \mathrm{x}_{\mathrm{S}}}\left[\mathcal{F}_{j} \mid \boldsymbol{Y}_{\mathrm{S}}=\boldsymbol{y}_{\mathrm{S}}\right] \\
& \mathbb{E}_{\boldsymbol{\Delta} \mathbf{X}_{\mathrm{S}}}\left[\mathcal{F}_{33} \mid \boldsymbol{Y}_{\mathrm{S}}=\boldsymbol{y}_{\mathrm{S}}\right]=\frac{1}{\sigma^{2}} \sum_{j=1}^{J} 2\left(l_{j}+1\right) \text {. }
\end{aligned}
$$

After obtaining the conditional expectation, the Fisher information $\mathbf{I}(\boldsymbol{\theta})$ can be evaluated by taking expectation with respect to $\boldsymbol{Y}_{\mathrm{S}}$. Unfortunately, it is difficult to express the distribution of $\boldsymbol{Y}_{\mathrm{S}}$ analytically, thus we employ a Monte Carlo integration method to compute the expectation based on the approximated conditional distributions. The details have been presented in Appendix B.

It is noted that the true parameter $\boldsymbol{\theta}$ cannot be revealed by the limited data, making it impossible to obtain the true Fisher information. Under a relatively large sample size, we can use the observed Fisher information to estimate the approximated distribution of the MLE, i.e., $\mathcal{N}\left(\boldsymbol{\theta},[\mathbf{I}(\hat{\boldsymbol{\theta}})]^{-\mathbf{1}}\right)$. Therefore, normal confidence intervals or confidence bands can be formulated for unknown parameters.

\section{Confidence Interval Construction With Bootstrapping Ap- proach}

Bootstrapping methods provide a resampling framework to numerically evaluate standard errors and confidence intervals, and it has been widely adopted in reliability analysis, especially when available data is limited. Bootstrapping confidence intervals are based on the normal approximation as well and they can provide comparisons with the results from Section C.

The procedures for constructing the $100(1-\alpha) \%$ confidence intervals with parametric bootstrapping are as follows:

1. Obtain the MLE $\hat{\boldsymbol{\theta}}=\left(\hat{\beta}_{0}, \hat{\beta}_{1}, \hat{\sigma}\right)^{\prime}$ from the observed data by the procedures described in Sections A-B.

2. Generate a bootstrap sample $\mathbf{B S}^{*}=\left(\boldsymbol{\Delta} \mathbf{x}^{*}, \boldsymbol{y}^{*}\right)$ with $\hat{\boldsymbol{\theta}}$. The details of the simulation are listed in Table I.
TABLE I

ALgorithm to SimUlate Data WITH GiVen PaRAMETERS

\begin{tabular}{l}
\hline \hline Algorithm: Simulation of load-sharing data with given parameters \\
\hline 1. Generate $J$ Wiener processes with parameters $\eta_{1}$ and $\sigma$, starting at \\
$t=0$. Set a small $\epsilon$ as the simulation step size of time. \\
2. For $j=1, \ldots, J$, do the following: \\
At each (following) time epoch $k \Delta \tau$, record the degradation meas- \\
urement for each component until the $j$ th failure occurs in the sys- \\
tem at $y_{j}$, which is also recorded. The degradation levels for the sur- \\
viving $J-j$ components are recorded and their afterward degrada- \\
tion processes are simulated by Wiener processes with parameters \\
$\eta_{j+1}$ and $\sigma$. \\
Collect all the data from Step 2 and a sample of $\Delta \mathbf{x}^{*}, \boldsymbol{y}^{*}$ can be eas- \\
ily obtained.
\end{tabular}

3. Obtain the bootstrap MLE from sample $\mathbf{B S}^{*}$, and denote the estimates by $\hat{\boldsymbol{\theta}}^{*}$.

4. $\quad$ Repeat Step 2 and 3 for $B$ times.

5. Order the $B$ estimates of $\boldsymbol{\theta}$ for each parameter in ascending order.

To deal with the bias induced by the parametric bootstrapping methods, we use the bias-corrected and accelerated (BCa) bootstrap to construct confidence intervals for $\boldsymbol{\theta}$ [33], [34]. Specifically, let $\theta_{i}$ be the $i$ th element of $\boldsymbol{\theta}$, and sort the sample for each parameter as $\left(\hat{\theta}_{i}^{*}{ }^{(1)}, \ldots, \hat{\theta}_{i}^{*}(B)\right)$ in ascending order. The $100(1-\alpha) \%$ confidence interval for $\theta_{i}$ is

$$
\left(\hat{\theta}_{i}^{* B P_{L}^{(i)}}, \hat{\theta}_{i}^{* B P_{U}^{(i)}}\right) \text { for } i=1,2 \text { and } 3,
$$

where

$$
\begin{gathered}
P_{L}^{(i)}=\Phi\left\{\hat{z}_{0 i}+\frac{\hat{z}_{0 i}+z_{\alpha / 2}}{1-\hat{\alpha}_{i}\left(\hat{z}_{0 i}+z_{\alpha / 2}\right)}\right\}, \\
P_{U}^{(i)}=\Phi\left\{\hat{z}_{0 i}+\frac{\hat{z}_{0 i}+z_{1-\alpha / 2}}{1-\hat{\alpha}_{i}\left(\hat{z}_{0 i}+z_{1-\alpha / 2}\right)}\right\},
\end{gathered}
$$

and

$$
\hat{z}_{0 i}=\Phi^{-1}\left\{\frac{\text { number of } \hat{\theta}_{i}^{*}{ }^{(b)}<\hat{\theta}_{i}}{B}\right\} \text {, for } b=1, \ldots, B
$$

Further,

$$
\left.\left.\hat{\alpha}_{i}=\frac{\sum_{b=1}^{B}\left(\theta_{i}^{(\cdot)}-\hat{\theta}_{i}^{*}(b)\right)^{3}}{6\left[\sum _ { b = 1 } ^ { B } \left(\hat{\theta}_{i}^{(\cdot)}-\hat{\theta}_{i}^{*}(b)\right.\right.}\right)^{2}\right]^{3 / 2},
$$

where

$$
\hat{\theta}_{i}^{(\cdot)}=\sum_{b=1}^{B} \widehat{\theta}_{i}^{(b)} / B
$$


TABLE II

BIAS AND STANDARD ERROR OF PARAMETER ESTIMATES

\begin{tabular}{|c|c|c|c|c|c|c|}
\hline \multirow{2}{*}{$(N, \Delta \tau)$} & \multicolumn{2}{|c|}{$\beta_{0}$} & \multicolumn{2}{|c|}{$\beta_{1}$} & \multicolumn{2}{|c|}{$\sigma$} \\
\hline & bias & $\mathrm{SE}$ & bias & $\mathrm{SE}$ & bias & $\mathrm{SE}$ \\
\hline$(5,0.005)$ & -0.0046 & 0.0949 & -0.0348 & 0.2164 & -0.0012 & 0.0051 \\
\hline$(5,0.01)$ & -0.0048 & 0.0936 & -0.0350 & 0.2126 & -0.0015 & 0.0071 \\
\hline$(5,0.05)$ & -0.0055 & 0.1041 & -0.1562 & 0.2574 & -0.0026 & 0.0152 \\
\hline$(10,0.005)$ & -0.0047 & 0.0663 & -0.0359 & 0.1506 & -0.0010 & 0.0036 \\
\hline$(10,0.01)$ & -0.0036 & 0.0664 & -0.0438 & 0.1524 & -0.0012 & 0.0050 \\
\hline$\left(\begin{array}{ll}10 & 0.05\end{array}\right)$ & -0.0052 & 0.0724 & -0.1558 & 0.2121 & -0.0015 & 0.0107 \\
\hline$(20,0.005)$ & -0.0037 & 0.0472 & -0.0396 & 0.1101 & -0.0009 & 0.0026 \\
\hline$(20,0.01)$ & -0.0038 & 0.0470 & -0.0449 & 0.1125 & -0.0011 & 0.0036 \\
\hline$(20,0.05)$ & -0.0048 & 0.0525 & -0.1525 & 0.1832 & -0.0012 & 0.0077 \\
\hline
\end{tabular}

Reliability characteristics, such as mean time to failure (MTTF) and median life, are functions of $\boldsymbol{\theta}$. Based on the invariance property of MLEs, the MLEs of reliability characteristics can simply be obtained through $\hat{\boldsymbol{\theta}}$. Thus, the same BCa technique can be applied to give the confidence intervals for maximum likelihood estimated functions of $\hat{\boldsymbol{\theta}}$.

\section{E. Discussions of Other Stochastic Processes}

Throughout the paper, we have assumed that the degradation is modeled by a Wiener process. In fact, numerous engineering experiences have revealed that many types of degradations are non-decreasing with respect to time, which cannot be properly characterized by a Wiener process. Gamma processes and inverse Gaussian processes are widely adopted both in the literature and in the application, and they can be alternatively used to model the load-sharing systems described in the paper. For both types of processes, the parameters can be obtained by using MLE via constructing the log-likelihood function as in (11). For readers of interest, we provide a brief description of the modeling problems under gamma and inverse Gaussian processes in Appendix D.

\section{SIMULATION STUDY}

To better illustrate the proposed parameter estimation framework, we carry out a comprehensive simulation study. Monte Carlo simulation is employed to generate random sample data for the load-sharing systems. Without any loss of generality, we assume that $J=4$. Following the example in Appendix A, the standardized stress is given by $\boldsymbol{\xi}=(0,0.208,0.5,1)^{\prime}$. By assuming the failure threshold to be 0.4 , we use the following parameter setting to simulate the data: $\boldsymbol{\theta}=\left(\beta_{0}, \beta_{1}, \sigma\right)^{\prime}=$ $(0.1,1,0.25)^{\prime}$. We explore the cases where $N=5,10$ and 20 to investigate the influence of sample sizes on the standard errors and bias. Under each sample size, we specify various inspection intervals: $\Delta \tau=0.005,0.01$ and 0.05 . Following the assumed true parameter, the MTTF and life median is 0.4247 and 0.4210 , respectively. By numerically simulating the failure time $T$, we plot the probability density and the reliability curves in Fig. 2. The density curve shows that the lifetime of the simulated system follows a right-skewed unimodal distribution.

\section{A. Estimation Bias and Standard Errors}

With 10,000 simulation replications, the mean bias and standard error (SE) of parameter estimates for each combination of $(N, \Delta \tau)$ are calculated and listed in Table II. The results show that, when $N$ increases, both the bias and standard error significantly reduce for all parameter estimates. By comparison, the influence of $\Delta \tau$ on the estimates of $\beta_{0}$ and $\beta_{1}$ is smaller than on the estimate of $\sigma$. We can observe that the biases and SEs under $\Delta \tau=0.005$ and 0.01 with a same $N$ are also relatively close. This implies that, regarding the accuracy of the estimates of $\beta_{0}$ and $\beta_{2}$, the influence of $\Delta \tau$ gets larger when $\Delta \tau$ is relatively large, whereas $N$ always puts a significant effect
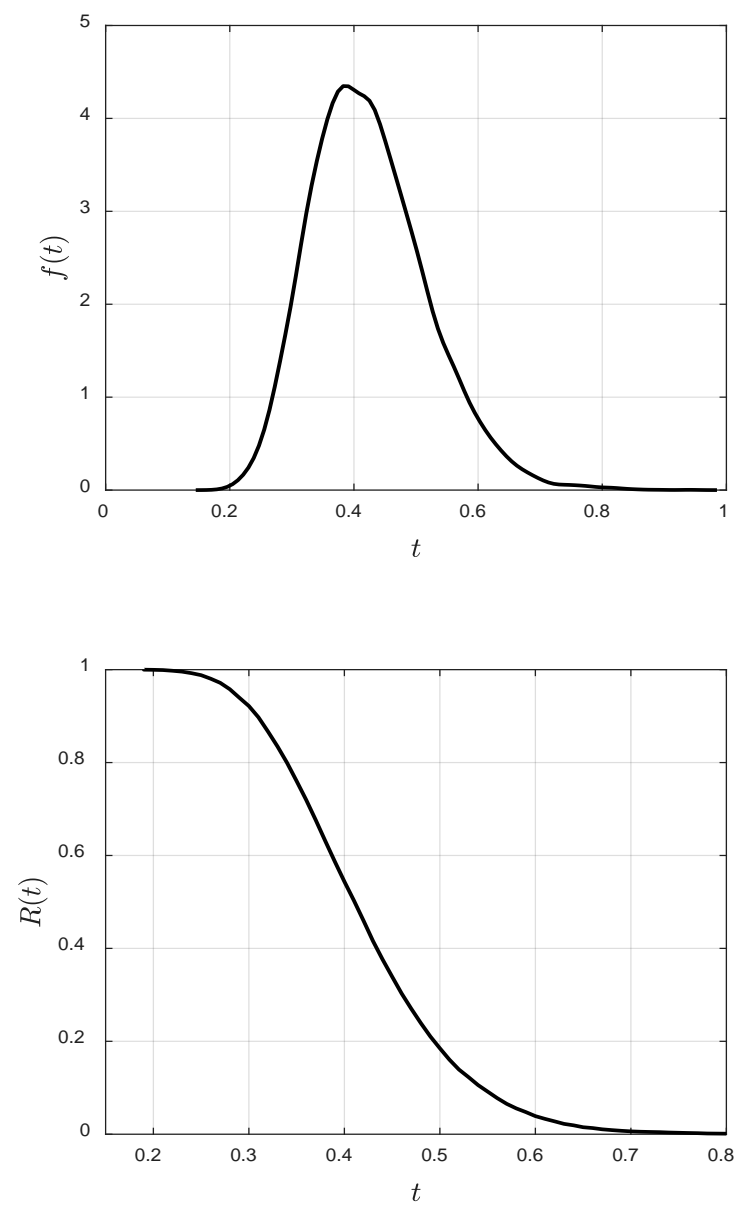

Fig. 2. Plots of failure time density and reliability 
TABLE III

COMPARISON OF COMPUTATIONAL EFFORTS

\begin{tabular}{cccc}
\hline \hline Initial point & $E\left(N_{I}\right)$ & $S D\left(N_{I}\right)$ & $E\left(T_{I}\right)$ \\
\hline Proposed guess & 14.2280 & 3.0612 & $0.4353 \mathrm{~s}$ \\
True value & 14.7600 & 3.1193 & $0.4656 \mathrm{~s}$ \\
$\boldsymbol{\theta}^{(0)}=(0,0,0.1)^{\prime}$ & 29.2500 & 4.5801 & $0.6203 \mathrm{~s}$ \\
$\boldsymbol{\theta}^{(0)}=(1,1,1)^{\prime}$ & 25.9820 & 3.0908 & $0.6223 \mathrm{~s}$ \\
\hline
\end{tabular}

on all the three estimates. The simulated example has followed mild and applicable assumptions on $(N, \Delta \tau)$. In other words, it is generally not very difficult to observe up to 20 test systems and measure the number of degradation measures for less than 100 times. When $\Delta \tau=0.05$, each component is only inspected less than 10 times. Under these assumptions, the bias and SE are still quite small compared to the absolute value of true parameters.

It is of interest to evaluate the required computational efforts to estimate the unknown parameters. For illustration, we select the case where $(N, \Delta \tau)=(10,0.01)$. Since we have used the "fminunc" function in MATLAB that utilizes the Quasi-Newton algorithm, the performance is considerably influenced by the initial point. We consider three types of initial points: (1) the proposed initial guess described in Section III.B; (2) The assumed true parameters; (3) Fixed pilot parameters. It is noted that the true parameters are in a black-box. We just carry out the analysis for illustration and it is impossible to reveal true parameters with limited data. Specifically, we set the initial point as presented in Table III and give the expected iteration number $E\left(N_{I}\right)$, standard deviation of the iteration number $S D\left(N_{I}\right)$ and the mean computing time $E\left(T_{I}\right)$ under 1,000 simulation runs for each case. Note that we use an Intel i5 core CPU @ 3.3GHz. The result shows the proposed initial guesses provide better computational efficiency with a lower $E\left(N_{I}\right)$, $S D\left(N_{I}\right)$ and $E\left(T_{I}\right)$ compared to the case where initial point starts at the true parameters. If the initial point is fixed pilot parameters that are different from the true ones, the computational efficiency will be even worse. The result implies that the proposed guessing method accelerates the estimation procedure via providing initial points close to the MLEs.

\section{B. Reliability Inferences}

Since reliability assessment is more intuitive and useful for practical purposes such as inspection and maintenance planning, we are also interested in how the uncertainty of estimated parameters affects the inference of system reliability. We choose the following three reliability related quantities to compare the simulated results: mean time to failure (MTTF), median life and the $10 \%$ life percentile $\left(T_{0.1}\right)$. The mean biases and SEs are summarized in Table IV. The pointwise 95\% confidence intervals of reliability curves are plotted in Fig. 3. The results are consistent with the those in the previous section. As $N$ increases from 5 to 10 , the pointwise confidence bands for the reliability curve get narrower significantly. Additionally, a smaller $\Delta \tau$ also helps to enhance the accuracy of reliability inference to a moderate extent. Specifically, under the same $N$, a small $\Delta \tau$ gives confidence intervals with lower and upper bounds that are relatively symmetric with respect to the true values, while for the cases with larger $\Delta \tau$, the upper bounds are further from the true values and the lower bounds are closer. This implies a larger bias in reliability inferences under parameter uncertainty if $\Delta \tau$ is large. Thus, more test systems enhance the estimation accuracy by reducing the uncertainty significantly, while smaller inspection intervals help more to reduce the bias of reliability inferences. It is noted that even under the case where $\Delta \tau=0.05$, the biases and SEs are not inflated drastically. They are acceptable if the engineers try to reduce cost with less inspections in real tests.

\section{AN APPLICATION EXAMPLE}

\section{A. Data Background}

The degradation of rail tracks can lead to serious safety problems in real operation [35]. Among various types of rail track degradation, the track geometry degradation is deemed to be one of the most important quality characteristics. The adjoining small sections are believed to share the loads from regularly operating trains. From the degradation test data where three short adjoining testing tracks (called by "components" afterward) as a system were put into continuous use. Seven systems of this type were tested at the same time, i.e., $N=7, J=3$. The data is plotted in Fig. 4 and each system is arranged in separate subplots. For confidentiality purposes, the data is transformed and truncated. By intuitively observing the degradation data, we

TABLE IV

BIAS AND STANDARD ERROR OF THREE COMMON RELIABILITY CHARACTERISTICS

\begin{tabular}{|c|c|c|c|c|c|c|}
\hline \multirow{2}{*}{$(N, \Delta \tau)$} & \multicolumn{2}{|c|}{ MTTF } & \multicolumn{2}{|c|}{ Median life } & \multicolumn{2}{|c|}{$T_{0.1}$} \\
\hline & bias & SE & bias & SE & bias & $\mathrm{SE}$ \\
\hline$(5,0.005)$ & -0.0244 & 0.0412 & -0.0197 & 0.0379 & 0.0006 & 0.0233 \\
\hline$(5,0.01)$ & -0.0317 & 0.0454 & -0.0265 & 0.0412 & -0.0040 & 0.0233 \\
\hline$(5,0.05)$ & -0.0344 & 0.0474 & -0.0291 & 0.0434 & -0.0061 & 0.0238 \\
\hline$(10,0.005)$ & -0.0257 & 0.0349 & -0.0211 & 0.0313 & -0.0006 & 0.0168 \\
\hline$(10,0.01)$ & -0.0336 & 0.0413 & -0.0283 & 0.0368 & -0.0052 & 0.0182 \\
\hline$\left(\begin{array}{ll}10 & 0.05\end{array}\right)$ & -0.0335 & 0.0406 & -0.0282 & 0.0362 & -0.0050 & 0.0173 \\
\hline$(20,0.005)$ & -0.0244 & 0.0299 & -0.0194 & 0.0259 & 0.0006 & 0.0126 \\
\hline$(20,0.01)$ & -0.0327 & 0.0366 & -0.0275 & 0.0321 & -0.0044 & 0.0129 \\
\hline$(20,0.05)$ & -0.0325 & 0.0362 & -0.0272 & 0.0316 & -0.0042 & 0.0125 \\
\hline
\end{tabular}



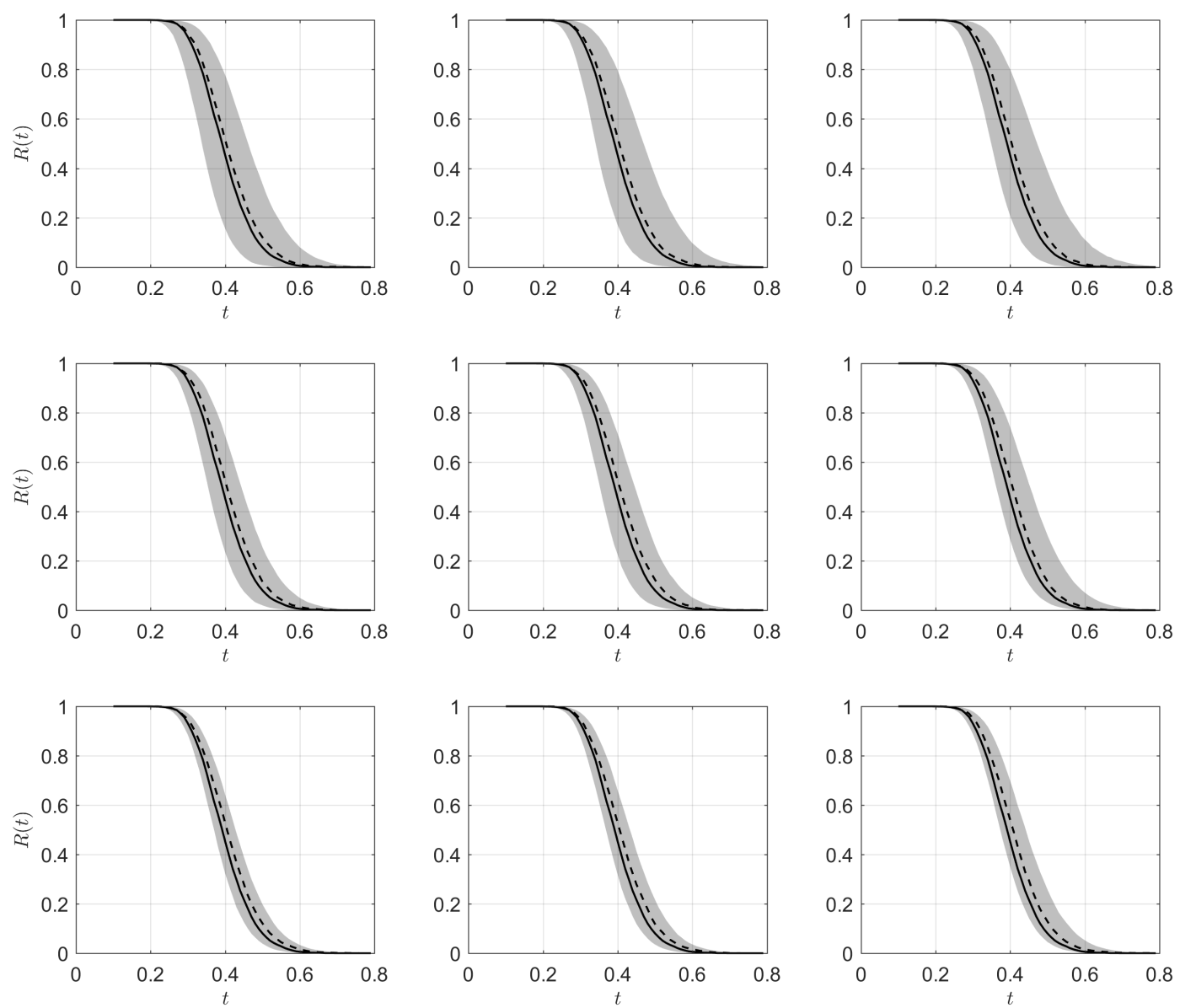

- Reliability at true $\theta$

Fig. 3. Reliability curves under $\boldsymbol{\theta}$ and mean $\hat{\boldsymbol{\theta}}$ under nine simulation cases. (Pointwise $95 \%$ confidence interval for $R(t)$ is shaded in each subplot)
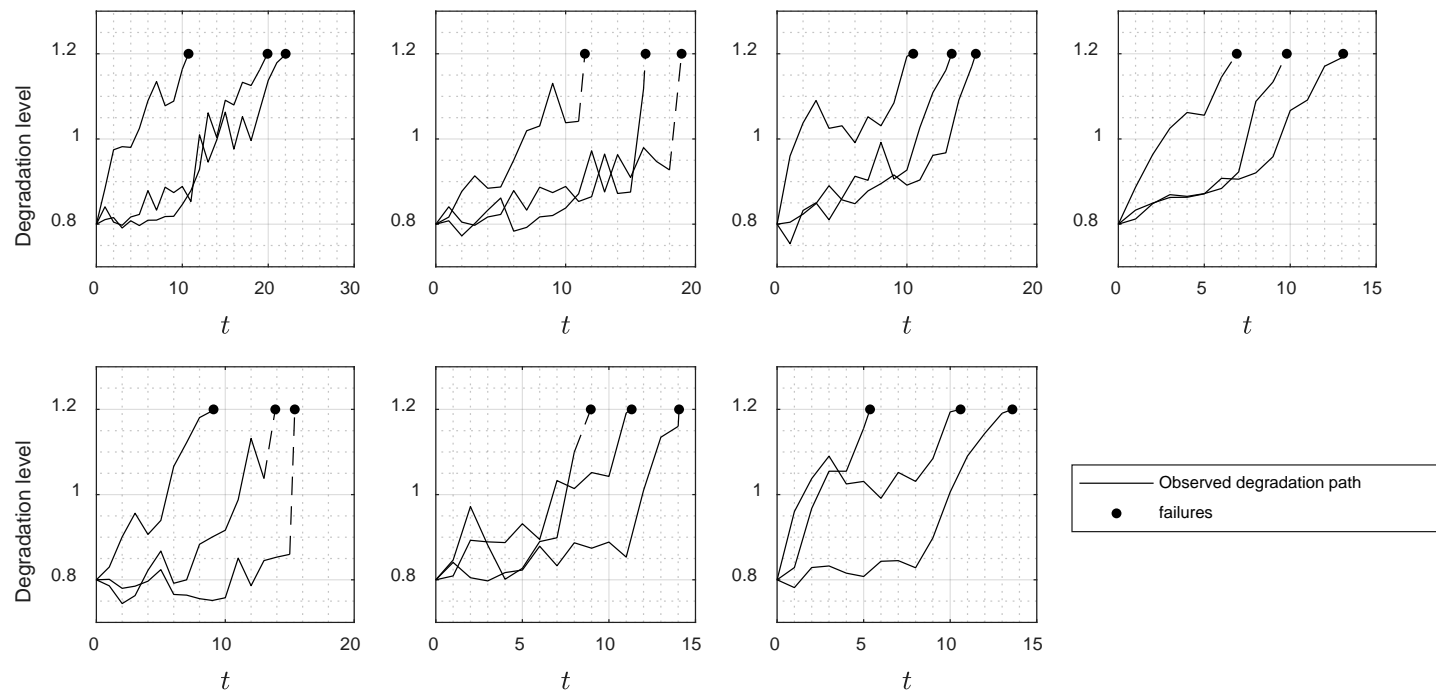

Fig. 4. Track geometry degradation testing data

find that the degradation rate after some component failures is higher than the degradation rate when the system initiates. Thus, we propose to fit the proposed model to the data. The degradation failure threshold is assumed to be 1.2 and all components initiates at level 0.8 , thus for further modeling, $L=$ $1.2-0.8=0.4$. For mathematical convenience, we simply set 

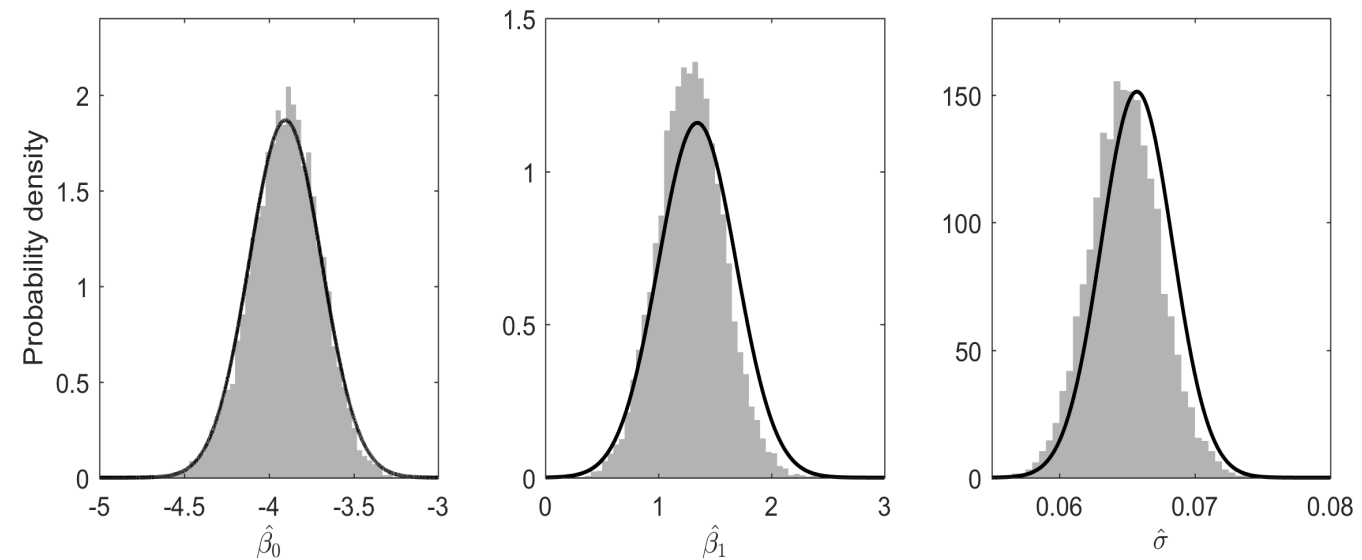

Fig. 5. Histograms of bootstrapping parameter samples vs. density curves of large-sample approximated normal distributions centered at MLE

TABLE V

MODEL COMPARISON BETWEEN MODELS WITH AND WITHOUT LINK FUNCTIONS

\begin{tabular}{|c|c|c|c|}
\hline Model & $\begin{array}{l}\text { Log-likeli- } \\
\text { hood }\end{array}$ & AIC & BIC \\
\hline $\begin{array}{l}\text { Proposed model with link func- } \\
\text { tion }\end{array}$ & 326.7415 & -647.48 & -636.57 \\
\hline $\begin{array}{l}\text { Separate parameters for each } \\
\text { component }\end{array}$ & 327.6709 & -647.34 & -632.79 \\
\hline
\end{tabular}

TABLE VI

STANDARD ERROR AND 90\% CONFIDENCE INTERVALS FOR $\boldsymbol{\theta}$ UNDER BS AND LS METHODS

\begin{tabular}{ccccccc}
\hline \hline & \multirow{2}{*}{ MLE } & \multicolumn{2}{c}{ Standard error } & & \multicolumn{2}{c}{$90 \%$ Confidence interval } \\
\cline { 3 - 4 } \cline { 5 - 6 } & & BS & LS & & BS & LS \\
\hline$\beta_{0}$ & -3.902 & 0.207 & 0.214 & & $(-4.272,-3.585)$ & $(-4.254,-3.551)$ \\
$\beta_{1}$ & 1.343 & 0.294 & 0.344 & & $(0.918,1.899)$ & $(0.777,1.901)$ \\
$\sigma$ & 0.066 & 0.003 & 0.003 & & $(0.062,0.071)$ & $(0.061,0.070)$ \\
\hline \hline
\end{tabular}

$\Delta \tau=1$. For the stress load, since the main reason of the degradation is the physical pressure on the track surface, thus we use a power law transformation [25] similar to the example used in Appendix A, which yields $\boldsymbol{\xi}=(0,0.6309,1)^{\prime}$.

\section{B. Parameter Estimation and Estimation Uncertainty}

By maximizing the log-likelihood, we obtain the MLE of unknown parameters $\quad \hat{\boldsymbol{\theta}}=\left(\hat{\beta}_{0}, \hat{\beta}_{1}, \hat{\sigma}\right)=$ $(-3.9024,1.3434,0.0657)^{\prime}$, under the maximized log-likelihood $\ell(\hat{\boldsymbol{\theta}} \mid \boldsymbol{\Delta x}, \boldsymbol{y})=326.7415$. Under the estimated parameters, the MTTF is 17.5038. The estimated degradation drift under the cases where the number of working components is 3,2 and 1 are $0.0202,0.0471$ and 0.0774 , respectively. The estimation results shed light to the fact that the change in degradation rate is considerable, as the degradation is several times faster when one or two components have failed in the system. We conduct a model comparison to show the performance of the proposed link function. In Liu et al. [23], parameters of the degradation model with different number of remaining components are regarded separate, and this increases the number of unknown parameters to be estimated. Under the proposed method and the model without link functions, we obtain the maximized log-likelihood and corresponding AIC and BIC for each model, as shown in Table $\mathrm{V}$. The proposed method gives smaller AIC and BIC, which implies that the link function has reasonable reduced the number of unknown parameters without much information loss to characterize the load-sharing degradation behaviors. Moreover, the estimated link function facilitates the extrapolated analysis if more components can be added to the system. To investigate the variability of parameter estimates, we use both the bootstrapping (BS) method and large-sample approximation (LS) to describe the MLEs as random variables. Note that we set the bootstrapping sample size $B=10,000$. The histograms of BS samples and LS approximated normal distributions are plotted in Fig. 5 for the unknown parameters. From the figures, the BS samples are roughly in consistence with the LS approximated distributions. Specifically, for $\beta_{0}$, the consistency is almost perfect, whereas for $\beta_{1}$ and $\sigma$, the modes of LS approximated distributions are slightly larger than those of BS samples.

Table VI lists the standard errors and 90\% confidence intervals (CI) for unknown parameters under BS and LS methods. The variability of $\beta_{1}$ is larger than that of $\beta_{0}$ for the reason that the number of degradation measurements after the first component failure is relatively small because the degradation rate increases fast as components fail one by one; additionally, because the number of components in this system is small $(J=$ 3 ), thus we can have at most three stress levels to estimate $\beta_{1}$, which also leads to a larger uncertainty in the inference of $\beta_{1}$. Nevertheless, the $90 \%$ CI shows that $\beta_{1}$ is significantly larger than 0 , and actually, based on our calculation, the lower bound of $95 \%$ CI is still larger than 0.65 . This again verifies the fact that the influence of component failure on the degradation rate is significant and positive. In other words, the trackway section degrades faster if the degradation levels of some subsections have reached the critical value.

\section{Reliability Inferences}

In Section IV.B, we computed three reliability characteristics for the simulated example. Likewise, we calculate the MLEs and $90 \%$ CIs for MTTF, median life and $T_{0.1}$ under BS and LS methods in Table VII. Furthermore, reliability curves under the MLE of $\boldsymbol{\theta}$ and mean $\hat{\boldsymbol{\theta}}$ under BS and LS method are shown in 

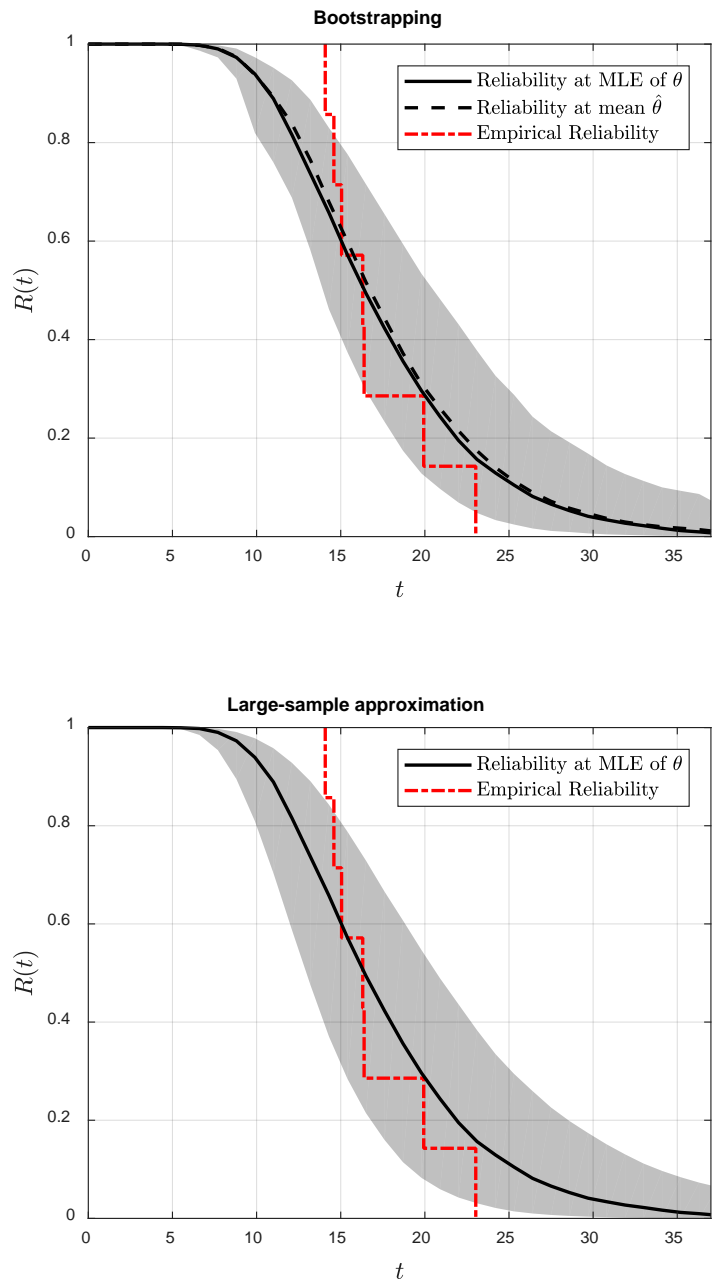

Fig. 6. Reliability curves under the MLE of $\boldsymbol{\theta}$ and mean $\hat{\boldsymbol{\theta}}$ under BS and LS method. (90\% pointwise confidence intervals for $\hat{\boldsymbol{\theta}}$ are shaded)

TABLE VII

STANDARD ERROR AND 90\% CONFIDENCE INTERVALS FOR THREE COMMON RELIABILITY CHARACTERISTICS UNDER BS AND LS METHODS

\begin{tabular}{cccc}
\hline \hline \multirow{2}{*}{ MLE } & \multicolumn{2}{c}{ 90\% Confidence interval } \\
\cline { 3 - 4 } & & BS & LS \\
\hline MTTF & 17.504 & $(13.432,20.392)$ & $(14.094,22.524)$ \\
Median life & 16.493 & $(12.124,18.689)$ & $(13.341,20.910)$ \\
$T_{0.1}$ & 10.800 & $(8.802,12.102)$ & $(8.791,13.212)$ \\
\hline \hline
\end{tabular}

Fig. 6. Pointwise $90 \%$ confidence bands for $R(t)$ and empirical reliability function are also plotted in the figure. For the tested system, the MLE of MTTF is slightly larger than median life, and this indicates the right skewness of the lifetime distribution. By comparison, the LS approximated CI is slightly wider than the CI calculated by BS samples.

From Fig. 6., the reliability curve evaluated at mean $\hat{\boldsymbol{\theta}}$ under BS method is very close to the curve at the point MLE. Moreover, the $90 \%$ confidence band for the reliability curve is wider under the LS approximation method. It is noted that the empirical reliability plot shows the sparsity of failure data because there are only 7 test systems. Under such cases, with only limited amount of failure data, it is challenging to use the existing life-based load-sharing models to obtain a reasonable interval inference for the parameters and reliability. By utilizing both the degradation measurements and soft failure time data, the proposed methods provide an approach to analyze load-sharing systems with degrading components similar to the system in the example. Given the estimated parameters and reliability function with the consideration of estimation uncertainty, decision makers can seek for more effective and robust monitoring and maintenance policies for such systems.

\section{CONCLUSIONS}

The paper has proposed a reliability modeling approach to degradation and life data obtained from parallel load-sharing systems. Analogous to the step-stress degradation models, the degradation rates of system components are assumed to increase according to a log-linear link function when components fail sequentially, which reduces the number of unknown parameters. Maximum likelihood estimation is adopted to estimate the parameters. Initial guesses are proposed to facilitate the estimation procedure. Regarding the estimation uncertainty, we use both bootstrapping and large-sample approximation methods to evaluate the variability of parameter estimates. Simulation methods are adopted to efficiently evaluate the reliability of the load-sharing systems. The simulation study shows an acceptable bias and standard error under a moderate assumption of sample sizes and inspection intervals. The whole framework is illustrated by an application example where track geometry degradation data are modeled and the reliability is assessed. The proposed systematic parameter estimation and reliability assessment methods can reasonably characterize load-sharing systems with degrading components and yield parameter and reliability estimates with the consideration of uncertainty, thus effectively support the decision making on system health management and maintenance.

Several related topics worth further investigation. First, the proposed methods can be extended to other types of load-sharing systems, such as $k$-out-of- $n$ systems and systems with nonidentical components. Another research direction is to generalize the load-sharing model. Random and cumulative loads as well as various load-sharing rules are of interest to study. The data modeling framework can be extended to deal with test data involving censoring. Additionally, as mentioned before, various management problems related to system reliability can be formulated for such systems, and to solve the optimization problem robustly by considering parameter uncertainty should be very useful for decision makers in various industries.

\section{APPENDIX}

\section{A. Discussions on the Link Function and $\xi\left(w_{j}\right)$}

First, we discuss the standardization of stress levels under various workloads based on the log-linear link function. Suppose that we know the relationship between the stress level $S_{j}$ and respective workload $w_{j}$, i.e., $S\left(w_{j}\right)$, then we can standardize the stress levels as follows for several common relations in accelerated degradation models: 
TABLE A.I

AN EXAMPLE OF VOLTAGE LOAD-SHARING SYSTEM

\begin{tabular}{llll}
\hline \hline $\begin{array}{l}\text { Surviving } \\
\text { components }\end{array}$ & Load & $\begin{array}{l}\text { Original } \\
\text { stress }\end{array}$ & $\begin{array}{l}\text { Standardized } \\
\text { stress }\end{array}$ \\
\hline 4 & $1 / 4$ & $0.75 \mathrm{kV}$ & 0 \\
3 & $1 / 3$ & $1 \mathrm{kV}$ & 0.208 \\
2 & $1 / 2$ & $1.5 \mathrm{kV}$ & 0.5 \\
1 & 1 & $3 \mathrm{kV}$ & 1 \\
\hline \hline
\end{tabular}

$$
\begin{array}{ll}
\xi_{j}=\frac{\ln S_{j}-\ln S_{0}}{\ln S_{H}-\ln S_{0}} & \text { for the power law relation } \\
=\frac{1 / S_{0}-1 / S_{j}}{1 / S_{0}-1 / S_{H}} & \text { for the Arrhenius relation } \\
=\frac{S_{j}-S_{0}}{S_{H}-S_{0}} & \text { for the exponential relation }
\end{array}
$$

For the load-sharing system we have assumed, it is also essential to identify $S\left(w_{j}\right)$, with which we can easily obtain $\xi\left(w_{j}\right)$ by standardization. $S\left(w_{j}\right)$ varies under different the failure mechanisms and accelerating factors. We use the voltage stress as an example. Suppose the system has 4 load-sharing components that evenly withstand a total $3 \mathrm{kV}$ of voltage. By a power law relation, we can compute the original and standardized stress levels on each component under the conditions where there are 4, 3, 2 and 1 surviving components as in Table A.I. We can see a difference between the workload and standardized stress, although they are both in $0 \sim 1$ scale. Thus, we cannot simply use the workload as the explanatory variable of the log-linear link function as it could lead to an unreasonable modeling of a known failure acceleration mechanism.

\section{B. Algorithm to Sample From $\boldsymbol{Y}_{S}$}

First, let $N_{\text {sim }}$ be the sample size of the simulation. We give the algorithm to generate one sample from $\boldsymbol{Y}_{i}$, denoted by $\boldsymbol{y}_{i}=$ $\left(y_{i, 1}, \ldots, y_{i, J}\right)^{\prime}$. To obtain a random sample of $y_{i, 1}$ is equivalent to draw a random sample from $Y_{1}$, of which the distribution can be described by

$$
\operatorname{Pr}\left(Y_{i, 1}>y\right)=\operatorname{Pr}\left(T_{i, 1}^{(1)}>y, T_{i, 2}^{(1)}>y, \ldots, T_{i, J}^{(1)}>y\right) .
$$

where $T_{i, 1}^{(1)}, \ldots, T_{i, J}^{(1)}$ follow independent and identical IG distributions denoted by $\mathcal{J} \mathcal{G}\left(L / \eta_{1}, L^{2} / \sigma^{2}\right)$. Thus,

$$
\begin{aligned}
\operatorname{Pr}\left(Y_{i, 1}>y\right)= & \operatorname{Pr}\left(T_{i, 1}^{(1)}>y\right) \ldots \operatorname{Pr}\left(T_{i, J}^{(1)}>y\right) \\
& =\prod_{j=1}^{J}\left(1-F_{\mathcal{J G}}\left(y ; L / \eta_{1}, L^{2} / \sigma^{2}\right)\right) .
\end{aligned}
$$

It is straightforward to draw one sample of $Y_{i, 1}$ by using the inverse transform sampling method. After obtaining $y_{i, 1}$, we an- alyze the possible conditions of the remaining $J-1$ components. Let $\boldsymbol{X}^{(1)}=\left(X_{1}^{(1)}, \ldots, X_{J-1}^{(1)}\right)^{\prime}$ be the vector of degradation levels of the remaining $J-1$ components for system $i$. Approximately, $X_{1}^{(1)}, \ldots, X_{J-1}^{(1)}$ are i.i.d. random variables that follow truncated normal distributions with mean $\eta_{1} y_{1}$, variance $y_{1} \sigma^{2}$, and upper bound $L$. The truncated distribution is denoted by $\mathcal{T} \mathcal{N}\left(\eta_{1} y_{1}, y_{1} \sigma^{2},-\infty, L\right)$. Afterward, we draw a sample from $\boldsymbol{X}^{(1)}$ based on the truncated distribution and have $\boldsymbol{x}^{(1)}=$ $\left(x_{1}^{(1)}, \ldots, x_{J-1}^{(1)}\right)^{\prime}$. Note that from time $y_{i, 1}$, we can assume that the Wiener degradation processes initials at $\boldsymbol{x}^{(1)}$ for the remaining components. Thus, the distribution of $Y_{i, 2}$ can be evaluated as:

$$
\begin{aligned}
\operatorname{Pr}\left(Y_{i, 2}>y\right)= & \operatorname{Pr}\left(T_{i, 1}^{(2)}>y, T_{i, 2}^{(2)}>y, \ldots, T_{i, J-1}^{(2)}>y\right) \\
& =\operatorname{Pr}\left(T_{i, 1}^{(2)}>y\right) \ldots \operatorname{Pr}\left(T_{i, J-1}^{(2)}>y\right), y \\
& \geq y_{i, 1},
\end{aligned}
$$

where $T_{i, j}^{(2)} \sim \mathcal{J} \mathcal{G}\left(\left(L-x_{j}^{(1)}\right) / \eta_{2},\left(L-x_{j}^{(1)}\right)^{2} / \sigma^{2}\right)$. It is also easy to draw a sample $y_{i, 2}$ from $Y_{i, 2}$. Similarly, for the remaining sampling for $y_{j}$, do the following step:

1. Draw $J-j+1$ samples from the following truncated distribution:

$$
\mathcal{T} \mathcal{N}\left(\sum_{j^{\prime}=1}^{j-1} \eta_{j^{\prime}} \Delta y_{j^{\prime}}, y_{j-1} \sigma^{2},-\infty, L\right)
$$

where $\Delta y_{j^{\prime}}=y_{j^{\prime}}-y_{j^{\prime}-1}$. Let $\boldsymbol{x}^{(j-1)}$ be the drawn samples.

2. Use the inverse sampling method to draw a $y_{j}$ by

$$
\begin{aligned}
\operatorname{Pr}\left(Y_{i, j}>y\right)= & \operatorname{Pr}\left(T_{i, 1}^{(j)}>y, \ldots, T_{i, J-j+1}^{(j)}>y\right) \\
& =\operatorname{Pr}\left(T_{i, 1}^{(j)}\right. \\
& >y) \ldots \operatorname{Pr}\left(T_{i, J-j+1}^{(j)}>y\right),
\end{aligned}
$$

where

$$
\begin{aligned}
& T_{i, j^{\prime}}^{(j)} \sim \mathcal{J} \mathcal{G}\left(\left(L-x_{j^{\prime}}^{(j-1)}\right) / \eta_{j},\left(L-x_{j^{\prime}}^{(j-1)}\right)^{2} / \sigma^{2}\right) . \\
& \text { For } j^{\prime}=1 \ldots, J-j+1 .
\end{aligned}
$$

3. End until $j=J$.

By replicating the above procedures, we can draw $N_{\text {sim }}$ samples from $\boldsymbol{Y}_{i}$. To be more accurate, one can resort to straightforward Monte Carlo simulation by generating Wiener processes. However, this could be much more computationally intense. 
The generated samples are then used to compute the expectation with respect to $\boldsymbol{Y}_{i}$, which is equivalent to $\boldsymbol{Y}_{\mathrm{S}}$ in (16). Monte Carlo integration can be used to compute the numerical results and obtain the Fisher information.

\section{Cases of Multiple Failures Occur in an Inspection Interval}

We discuss how to evaluate the likelihood contributions of the observations between two consecutive inspections if more than one failure occurs in the interval.

Upon the $(k-1)$ th inspection, we assume that $j^{\prime}-2$ components have failed, where $j^{\prime} \geq 2$. Moreover, $f \geq 2$ failures occur between the $k-1$ and $k$ th inspection. In other words, $L_{i\left(j^{\prime}+l-2\right)}=k-1$ for $l=1, \ldots, f$. For the surviving components upon $k$ th inspection, i.e., $j=j^{\prime}+k-1, \ldots, J$, the degradation increments follow the following normal distribution:

$$
\begin{array}{r}
\Delta X_{i j k} \sim \mathcal{N}\left(M_{i\left(j^{\prime}-1\right)} \eta_{j^{\prime}-1}+\sum_{l=j^{\prime}}^{j^{\prime}+f-2}\left(M_{i l}-M_{i(l-1)}\right) \eta_{l}\right. \\
\left.+\eta_{j^{\prime}+f-1}\left(\Delta \tau-M_{i\left(j^{\prime}+f-2\right)}\right), \sigma^{2} \Delta \tau\right) .
\end{array}
$$

Note that if there is no surviving component upon the $k$ th inspection, the degradation increments are unobservable thus cannot contribute to the likelihood function. Further, to model the random variables $M_{i\left(j^{\prime}-1\right)}, \ldots, M_{i\left(j^{\prime}+f-2\right)}$ by given realizations of the degradation levels from the last inspection $X_{i\left(j^{\prime}-1\right)(k-1)}=x_{j^{\prime}-1}, \ldots, X_{i\left(j^{\prime}+f-2\right)(k-1)}=x_{j^{\prime}+f-2}$, we can construct the likelihood contributions by $M_{i\left(j^{\prime}-1\right)}, \ldots, M_{i\left(j^{\prime}+f-2\right)}$, denoted by $\mathcal{L}^{\prime}$ and shown as follows:

$$
\begin{aligned}
& \mathcal{L}^{\prime}=f_{M_{i\left(j^{\prime}-1\right)}, \ldots, M_{i\left(j^{\prime}+f-2\right)}}\left(m_{j^{\prime}-1}, \ldots, m_{j^{\prime}+f-2}\right) \\
& =f_{M_{i\left(j^{\prime}-1\right)}}\left(m_{j^{\prime}-1}\right) \times f_{M_{i j^{\prime}} \mid M_{i\left(j^{\prime}-1\right)}}\left(m_{j^{\prime}} \mid m_{j^{\prime}-1}\right) \times \ldots \\
& \times f_{M_{i\left(j^{\prime}+f-2\right)} \mid M_{i\left(j^{\prime}-1\right)}, . ., M_{i\left(j^{\prime}+f-3\right)}}\left(m_{j^{\prime}+f-2} \mid m_{j^{\prime}-1}, \ldots, m_{j^{\prime}+f-3}\right),
\end{aligned}
$$

where following [36]:

$M_{i\left(j^{\prime}-1\right)} \sim \mathcal{J} \mathcal{G}\left(\frac{L-x_{j^{\prime}-1}}{\eta_{j^{\prime}-1}}, \frac{\left(L-x_{j^{\prime}-1}\right)^{2}}{\sigma^{2}}\right)$,

$M_{i j^{\prime}} \mid\left(M_{i\left(j^{\prime}-1\right)}\right.$

$\left.=m_{j^{\prime}-1}\right) \sim \mathcal{J G}\left(\begin{array}{c}\frac{L-x_{j^{\prime}}-\left(\eta_{j^{\prime}-1}-\eta_{j^{\prime}}\right) m_{j^{\prime}-1}}{\eta_{j^{\prime}}}, \\ \frac{\left[L-x_{j^{\prime}}-\left(\eta_{j^{\prime}-1}-\eta_{j^{\prime}}\right) m_{j^{\prime}-1}\right]^{2}}{\sigma^{2}}\end{array}\right)$,

$M_{i\left(j^{\prime}+f-2\right)} \mid\left(M_{i\left(j^{\prime}-1\right)}=m_{j^{\prime}-1}, \ldots, M_{i\left(j^{\prime}+f-3\right)}\right.$

$$
\left.=m_{j^{\prime}+f-3}\right) \sim \mathcal{J} \mathcal{G}(A, B),
$$

where

$$
\begin{gathered}
A=\frac{L-x_{j^{\prime}+f-2}+m_{i\left(j^{\prime}+f-3\right)} \eta_{j^{\prime}+f-2}}{\eta_{j^{\prime}+f-2}} \\
-\frac{\sum_{l=j^{\prime}-1}^{j^{\prime}+f-3}\left(m_{i l}-m_{i(l-1)}\right) \eta_{l}}{\eta_{j^{\prime}+f-2}}, \\
B=\frac{C^{2}}{\sigma^{2}}, \\
C=L-x_{j^{\prime}+f-2}+m_{i\left(j^{\prime}+f-3\right)} \eta_{j^{\prime}+f-2} \\
-\sum_{l=j^{\prime}-1}^{j^{\prime}+f-3}\left(m_{i l}-m_{i(l-1)}\right) \eta_{l},
\end{gathered}
$$

and $m_{i\left(j^{\prime}-2\right)} \equiv 0$. In this manner, the likelihood contributions from observations in an inspection interval in which multiple failures occur can be evaluated.

\section{Discussions for Gamma Process and IG Process}

Gamma process and inverse Gaussian (IG) process are commonly used to model monotone degradation path. Although they are inappropriate to model the data from the example in Section $\mathrm{V}$ as the degradation is non-monotone, they have been successful to model degradation data in many applications. First, we discuss the gamma process with shape and scale parameter given by $\alpha_{j}$ and $\gamma$, respectively. Similar to (2), the loglinear link function can be defined as

$$
\log \left(\alpha_{j}\right)=\beta_{0}+\beta_{1} \xi_{j}, \quad j=1, \ldots, J
$$

In other words, the scale parameter is assumed to be constant. Because gamma process is a pure jump process, the following reliability analysis is different from the Wiener process because of "overshoot behavior" [37]. To evaluate the time between two given degradation levels of gamma process, approximated methods are often resorted to.

For the IG process, Ye et al. [38, Sec. II. B] presented two possible schemes to connect the parameters of the IG process and the load stresses. First, we can assume the mean parameter $\log \left(\mu_{j}\right)=\beta_{0}+\beta_{1} \xi_{j}$ and keep the shape parameter $\lambda$ constant, and this makes both the degradation rate and volatility inflated with higher stress. Another scheme is to assume $\log \left(\lambda_{j}\right)=$ $\beta_{0}+\beta_{1} \xi_{j}$ and keep $\mu$ constant, therefore the stress does not influence the degradation rate, but increases the volatility of degradation paths. For the load-sharing systems discussed in the paper, it is more reasonable to adopt the earlier scheme. The following parameter estimation and reliability assessment can be carried based on the ideas in the paper as the pdf and cdf of the first-passage time of the IG process have been given in analytical forms in related studies [18]. Note that to derive the estimated distribution for unknown parameters may be tedious and trivial for the gamma and IG processes.

\section{REFERENCES}

[1] W. Kuo and M. J. Zuo, Optimal reliability modeling: principles and applications. John Wiley \& Sons, 2003.

[2] A. Bodas and A. Kahraman, "Influence of carrier and gear manufacturing errors on the static load sharing behavior of planetary gear Sets," JSME 
International Journal Series C, vol. 47, no. 3, pp. 908-915, 2004.

[3] M. Li, L. Xie, and L. Ding, "Load sharing analysis and reliability prediction for planetary gear train of helicopter," Mechanism and Machine Theory, vol. 115, pp. 97-113, 2017.

[4] J. Shao and L. R. Lamberson, "Modeling a shared-load k-out-of- $n: G$ system," IEEE Transactions on Reliability, vol. 40, no. 2, pp. 205-209, 1991.

[5] H. Kim and P. H. Kvam, "Reliability estimation based on system Data with an unknown load share rule," Lifetime Data Analysis, vol. 10, no. 1, pp. 83-94, 2004.

[6] B. Singh and P. K. Gupta, "Load-sharing system model and its application to the real data set," Mathematics and Computers in Simulation, vol. 82, no. 9, pp. 1615-1629, 2012.

[7] Y. Kong and Z. Ye, "Interval estimation for k-out-of-n load-sharing systems,” IISE Transactions, vol. 49, no. 3, pp. 344-353, 2017.

[8] S. Taghipour and M. L. Kassaei, "Periodic inspection optimization of a $k$ out-of-n load-sharing system," IEEE Transactions on Reliability, vol. 64, no. 3, pp. 1116-1127, 2015.

[9] W. Y. Yun, G. R. Kim, and H. Yamamoto, "Economic design of a loadsharing consecutive $k$-out-of- $n$ : F system," IIE Transactions, vol. 44, no. 1, pp. 55-67, 2012.

[10] H. Liu, "Reliability of a load-sharing k-out-of-n: G system: non-iid components with arbitrary distributions," IEEE Transactions on Reliability, vol. 47, no. 3, pp. 279-284, 1998.

[11] P. H. Kvam and E. A. Peña, "Estimating load-sharing properties in a dynamic reliability system," Journal of the American Statistical Association, vol. 100, no. 469, pp. 262-272, 2005.

[12] C. Park, "Parameter estimation for the reliability of load-sharing systems," IIE Transactions, vol. 42, no. 10, pp. 753-765, 2010.

[13] C. Park, "Parameter estimation from load-sharing system data using the expectation-maximization algorithm," IIE Transactions, vol. 45, no. 2, pp. 147-163, 2013.

[14] L. Wang, J. Zhang, W. Chen, and X. Jia, "Reliability evaluation of a loadsharing parallel system with failure dependence," Communications in Statistics - Simulation and Computation, vol. 45, no. 9, pp. 3094-3113, 2016.

[15] Z.-S. Ye, Y. Wang, K.-L. Tsui, and M. Pecht, "Degradation data analysis using Wiener processes with measurement errors," IEEE Transactions on Reliability, vol. 62, no. 4, pp. 772-780, 2013.

[16] Q. Zhai and Z.-S. Ye, "RUL prediction of deteriorating products using an adaptive Wiener process model,” IEEE Transactions on Industrial Informatics, vol. 13, no. 6, pp. 2911-2921, 2017.

[17] C. Meier-Hirmer, G. Riboulet, F. Sourget, and M. Roussignol, "Maintenance optimization for a system with a gamma deterioration process and intervention delay: application to track maintenance," Proceedings of the Institution of Mechanical Engineers, Part O: Journal of Risk and Reliability, vol. 223, no. 3, pp. 189-198, 2009.

[18] Z.-S. Ye and N. Chen, "The inverse Gaussian process as a degradation model,” Technometrics, vol. 56, no. 3, pp. 302-311, 2014.

[19] Y. Hong, Y. Duan, W. Q. Meeker, D. L. Stanley, and X. Gu, "Statistical methods for degradation data with dynamic covariates information and an application to outdoor weathering data," Technometrics, vol. 57, no. 2, pp. 180-193, 2015.

[20] Z. Xu, Y. Hong, and R. Jin, "Nonlinear general path models for degradation data with dynamic covariates," Applied Stochastic Models in Business and Industry, vol. 32, no. 2, pp. 153-167, 2016.

[21] Z. Ye, M. Revie, and L. Walls, "A load sharing system reliability model with managed component degradation," IEEE Transactions on Reliability, vol. 63, no. 3, pp. 721-730, 2014.

[22] B. Liu, M. Xie, and W. Kuo, "Reliability modeling and preventive maintenance of load-sharing systemswith degrading components," IIE Transactions, vol. 48, no. 8, pp. 699-709, 2016.

[23] B. Liu, J. Xu, and X. Zhao, "Parameter estimation for load-sharing systems with degrading components," in 2016 IEEE International Conference on Industrial Engineering and Engineering Management (IEEM), 2016, pp. 1310-1314.

[24] Y. Liu, Y. Pan, Z. Sun, and D. Huang, "Statistical monitoring of wastewater treatment plants using variational Bayesian PCA,” Industrial \& Engineering Chemistry Research, vol. 53, no. 8, pp. 3272-3282, 2014.

[25] W. Q. Meeker and L. A. Escobar, Statistical Methods for Reliability Data. John Wiley \& Sons, 1998.

[26] Y. Kong and Z.-S. Ye, "A cumulative-exposure-based algorithm for failure data from a load-sharing system," IEEE Transactions on Reliability, vol. 65, no. 2, pp. 1001-1013, 2016.

[27] F. Jakob, M. Kimmelmann, and B. Bertsche, "Selection of acceleration models for test planning and model usage," IEEE Transactions on Reliability, vol. 66, no. 2, pp. 298-308, 2017.

[28] S. Tseng and Z. Wen, "Step-stress accelerated degradation analysis for highly reliable products," Journal of Quality Technology, vol. 32, no. 3, pp. 209-216, 2000.

[29] C.-M. Liao and S.-T. Tseng, "Optimal design for step-stress accelerated degradation tests," IEEE Transactions on Reliability, vol. 55, no. 1, pp. 59-66, 2006.

[30] X. Zhao, J. Xu, and B. Liu, "Accelerated degradation tests planning with competing failure modes," IEEE Transactions on Reliability, vol. 67, no. 1, pp. 142-155, 2018.

[31] X. Zhao and M. Xie, "Using accelerated life tests data to predict warranty cost under imperfect repair," Computers \& Industrial Engineering, vol. 107, pp. 223-234, 2017.

[32] D. P. Bertsekas, Nonlinear programming. Athena Scientific Belmont, 1999.

[33] L. Shen, D. Sun, Z. Ye, and X. Zhao, "Inference on an adaptive accelerated life test with application to smart- grid data-acquisition-devices," Journal of Quality Technology, vol. 49, no. 3, pp. 191-212, 2017.

[34] G. Wang, Z. He, L. Xue, Q. Cui, S. Lv, and P. Zhou, "Bootstrap analysis of designed experiments for reliability improvement with a non-constant scale parameter," Reliability Engineering \& System Safety, vol. 160, pp. 114-121, 2017.

[35] I. Soleimanmeigouni, X. Xiao, A. Ahmadi, M. Xie, A. Nissen, and U. Kumar, "Modelling the evolution of ballasted railway track geometry by a two-level piecewise model," Structure and Infrastructure Engineering, vol. 14, no. 1, pp. 33-45, 2018.

[36] P. Wang, Y. Tang, S. Joo Bae, and Y. He, "Bayesian analysis of two-phase degradation data based on change-point Wiener process," Reliability Engineering \& System Safety, vol. 170, pp. 244-256, 2018.

[37] B. de Jonge, R. Teunter, and T. Tinga, "The influence of practical factors on the benefits of condition-based maintenance over time-based maintenance," Reliability Engineering \& System Safety, vol. 158, pp. 2130, 2017.

[38] Z. Ye, L. Chen, L. C. Tang, and M. Xie, "Accelerated degradation test planning using the inverse Gaussian process," IEEE Transactions on Reliability, vol. 63, no. 3, pp. 750-763, 2014.

Xiujie Zhao received the B.E. degree in industrial engineering from Tsinghua University, Beijing, China, in 2013, and the M.S. degree in industrial engineering from Pennsylvania State University, University Park, PA, USA, in 2015. He is currently working toward the Ph.D. degree in industrial engineering in the Department of Systems Engineering and Engineering Management, City University of Hong Kong, Hong Kong.

His research interests include accelerated reliability testing, degradation modeling, maintenance modeling, and design of experiments.

Bin Liu received the Ph.D. degree in industrial engineering from City University of Hong Kong, Hong Kong, in 2017, and the B.S. degree in automation from Zhejiang University, Zhejiang, China, in 2013.

He is currently a postdoctoral fellow with the Department of Civil and Environmental Engineering, University of Waterloo, ON, Canada. His research interests include reliability and maintenance modeling, importance measures with application to complex systems, and data analysis.

Yiqi Liu received the B.S. and M.S. degrees in control engineering from the Chemical University of Technology, Beijing, in 2009 and the Ph.D. degree in control engineering from South China University of Technology, Guangzhou, China, in 2013.

From 2013 to 2016, he was a Lecturer with the Department of Automation, South China University of Technology. Since 2016, he has been an Associate Professor with the same department. He is the author of more than 44 articles. His research interests include soft-sensors, fault diagnosis, and wastewater treatment and he holds three patents. He was a recipient of the Hongkong Scholar Award in 2016, Chinese Scholarship Council Award in 2011, and the Deutscher Akademischer Austausch Dienst Award in 2011. 